DOI: $10.1515 /$ awutm -2015-0006

DE GRUYTER
Analele Universitătii de Vest,

Timişoara

Seria Matematică - Informatică

LIII, 1, (2015), 109- 136

\title{
Systèmes dynamiques algébriquement complètement intégrables et géométrie
}

\author{
A. Lesfari
}

Résumé. In this paper I present the basic ideas and properties of the complex algebraic completely integrable dynamical systems. These are integrable systems whose trajectories are straight line motions on complex algebraic tori (abelian varieties). We make, via the Kowalewski-Painlevé analysis, a detailed study of the level manifolds of the system. These manifolds are described explicitly as being affine part of complex algebraic tori and the flow can be solved by quadrature, that is to say their solutions can be expressed in terms of abelian integrals. The Adler-van Moerbeke method's which will be used is primarily analytical but heavily inspired by algebraic geometrical methods. We will also discuss several examples of algebraic completely integrable systems : Kowalewski's top, geodesic flow on $S O(4)$, Hénon-Heiles system, Garnier potential, two coupled nonlinear Schrödinger equations and Yang-Mills system.

AMS Subject Classification (2000). 70H06, 14H70, 14J40, 14E25, 32Q15.

Keywords. Integrable systems, Riemann surfaces, Abelian varieties, Jacobian varieties, Prym varieties, Embeddings, Kähler manifolds. 


\section{Introduction et motivation}

La théorie des équations différentielles utilise actuellement un arsenal très riche d'idées et de méthodes mathématiques. En particulier et comme en témoignent plusieurs travaux récents, le grand intérêt porté ces dernières années sur l'étude des systèmes dynamiques complètement intégrables laisse à penser qu'un pont nouveau va être jeté entre les applications et les théories mathématiques abstraites les plus avancées. Il est bien connu qu'il n'est pas de question plus intéressante pour les applications que l'étude des équations différentielles. L'intérêt et la difficulté concernant ce domaine sont dus au fait que les équations différentielles décrivant la nature sont presque toutes non-linéaires. La solution des problèmes dynamiques non-linéaires par quadratures est généralement impossible et une solution numérique ne montre pas leurs propriétés qualitatives. Cependant, jusqu'à récemment, mathématiciens et physiciens durent se contenter, soit d'approximations linéaires, soit de théorèmes d'existence de solutions. La découverte ces dernières années de classes importantes de systèmes intégrables montra combien de phénomènes importants n'ont pas pu être découverts lors de l'étude de ces équations par des méthodes linéaires. En fait depuis longtemps, on avait utilisé dans tous les domaines les concepts de linéarisation et de superposition des modes. Les non-linéarités n'étaient traitées que comme des petites perturbations. Elles sont dorénavant examinés dans leur intégralité. Les premières études furent motivées par des problèmes de physique des plasmas, par la dynamique des fluides ou des chaînes. Citons à titre d'exemple l'équation de Korteweg-de-Vries [13] (et les références incluses) qui décrit la propagation des ondes dans un canal infini peu profond. Cette équation est caractérisée par un terme tendant à disperser l'onde et un autre terme non-linéaire ajusté de façon à contrebalancer les effets de dispersion. Un milieu dans lequel ces deux efforts se compensent sera propice à l'existence d'ondes solitaires ou solitons. Ce sont des ondes de formes définies progressant à des vitesses différentes et dont le profil est stable au cours de la propagation, par suite de cette compétition entre l'effet dispersif et l'effet non-linéaire. Généralement, on regroupe sous le vocable soliton des solutions d'équations d'ondes non-linéaires présentant les propriétés caractéristiques suivantes : elles sont localisées dans l'espace, durent indéfiniment et conservent leur amplitude et leur vitesse même à l'issue de plusieurs collisions avec d'autres solitons. En outre, ces ondes correspondent aux niveaux d'énergie de l'équation stationnaire de Schrödinger, ce qui a suggéré l'idée d'utiliser l'analogie avec la mécanique quantique. Et effectivement, la découverte de ce lien influença de nombreux domaines, des mathématiques à la technologie en passant par 
la physique et la biologie. A part l'équation de Korteweg-de-Vries, on peut citer parmi les équations non-linéaires ayant des solutions de type soliton, l'équation de Schrödinger non-linéaire qui gouverne la propagation d'une onde lumineuse dans une fibre optique dont le diamètre est faible comparé à la longueur d'onde, une autre équation aux dérivées partielles non-linéaire régit le transfert d'énergie (par hydrolyse d'adénosine triphosphate en adénosine diphosphate) le long des chaînes de protéines dans les organismes vivants. Entretemps, le sujet a évolué vers des thèmes plus mathématiques, liés aux algèbres de Lie et à la géométrie algébrique, en vue de répondre aux problèmes fondamentaux concernant la nature des équations différentielles ordinaires et équations aux dérivées partielles complètement intégrables. En particulier un nombre considérable de ces équations est entré dans le cadre de la mécanique hamiltonienne et possèdent plusieurs, voir même une infinité, de lois de conservation, en plus de la conservation de l'énergie et du moment. Ces lois résultent parfois de symétries apparentes au niveau de l'espace géométrique des configurations, parfois de symétries cachées au sein de l'espace des phases. On sait actuellement que ces symétries cachées, plus difficiles à saisir, se traduisent naturellement en termes de la théorie des groupes de Lie, qui systématise par excellence l'étude des symétries et qui établit le lien avec la théorie spectrale. A leur tour, ces groupes de Lie donnent lieu à des courbes algébriques ou surfaces de Riemann, d'où résultent des tores, ce qui nous conduit au coeur même de la géométrie algébrique. Ces tores jouent un rôle prépondérant car les trajectoires des systèmes mentionnés précédemment, peuvent être toutes considérées comme étant enroulées sur ces tores. La solution de ces problèmes de mécanique a donné lieu à un foisonnement d'idées et de liens entre les domaines apparemment les plus distants, comme la théorie spectrale, la géométrie algébrique et la théorie des groupes de Lie. En retour, cette synthèse alimenta chacun de ces domaines en idées nouvelles. Tout chercheur ayant participé aux rencontres scientifiques de ces dernières années, où expérimentateurs, géomètres et algébristes se sont côtoyés, aura été frappé par l'énorme diversité de sujets. Actuellement, les applications de la théorie des systèmes complètement intégrables sont nombreuses, notamment en physique des particules, en dynamique des plasmas et des fluides, en mécanique statistique, en biologie de fibres. La théorie des solitons joue un rôle important dans le domaine des télécommunications. Des signaux lumineux (solitons) ultra-courts envoyés dans certaines fibres optiques faites d'un matériau bien précis, peuvent voyager plus longtemps sans être brouillés et sans perdre l'information qu'ils transmettent. La construction de mémoires à temps de communication ultra-rapide et à faible consommation d'énergie (donc moins coûteuse pour l'internet, par exemple), est basée sur le mouvement de tourbillons magnétiques dans la jonction diélectrique qui sépare 
deux supraconducteurs. Au niveau moléculaire, la théorie des solitons permet d'élucider le mécanisme de contraction des muscles striés. Dans la chaîne de peptides (molécules constituées par l'union d'un petit nombre de molécules d'acides aminés) et d'hydrogène des protéines, les solitons naissent du mariage de la dispersion due aux vibrations intra-peptides et de la non-linéarité due à l'interaction de ces vibrations avec les déplacements de groupes peptides autour de leur position d'équilibre. Mais aussi la théorie des solitons a eu un impact sur les mathématiques pures; par exemple, il fournit la réponse au fameux problème de Schottky, posé il y a un siècle, sur les relations entre les périodes provenant d'une surface de Riemann. La théorie des systèmes algébriquement complètement intégrables [3] présente une stratégie de résolution d'une classe importante d'équations différentielles non-linéaires. Plus précisément, il vise une approche systématique dans la détection et la linéarisation des équations différentielles non-linéaires algébriquement intégrables. Les méthodes utilisées sont avant tout analytiques, mais très inspirées par des méthodes de géométrie complexe contemporaine. Quel est l'intérêt d'une telle approche? D'abord, il est global et est donc invariant pour tout changement de coordonnées. Ensuite, les méthodes utilisées sont directes, puissantes et permettent d'obtenir des résultas précis sur le comportement des solutions. Dans la section 2, je donne tout d'abord quelques notions et propriétés générales sur les systèmes algébriquement complètement intégrables. La section 3, est consacrée à l'étude des variétés invariantes, à la construction des tores complexes algébriques (variétés abéliennes) ainsi qu'à la linéarisation des systèmes algébriquement complètement intégrables. Nous verrons, dans cette section, que les solutions méromorphes dépendant d'un nombre suffisant de paramètres libres jouent un rôle crucial dans l'étude des équations différentielles dites algébriquement intégrables. Cela veut dire que l'on demande que les invariants du système différentiel soient polynomiaux (dans des coordonnés adéquates) et que de plus les variétés complexes obtenues en égalant ces invariants polynomiaux à des constantes génériques forment la partie affine d'une variété abélienne de telle façon que les flots complexes engendrés par les invariants du système soient des lignes droites sur ces tores complexes. Indépendamment du fait que la plupart des exemples classiques et nouveaux de systèmes hamiltoniens complètement intégrables sont algébriquement complètement intégrables, une motivation plus profonde pour leur étude est la suivante : ces systèmes apparaissent systématiquement lorsque l'on étudie les déformations isospectrales d'opérateurs linéaires contenant une indéterminée rationnelle (en bref : paire de Lax). En fait, un théorème de Adler-KostantSymes [3] appliqué aux algèbres de Kac-Moody (extensions formelles de dimension infinie d'une algèbre de Lie semi-simple) fournit de tels systèmes qui sont des déformations isospectrales et qui, par un théorème de van Moerbeke- 
Mumford [16], sont algébriquement complètement intégrables. Du point de vue de la mécanique, cela veut dire que les symétries cachées de beaucoup de systèmes algébriquement complètement intégrables s'expliquent par la théorie des groupes. Les méthodes utilisées sont avant tout analytiques, mais très inspirées par des méthodes de géométrie contemporaine. Dans la section 4, on expose quelques systèmes dynamiques non-linéaires importants, algébriquement complètement intégrables. On considère tout d'abord le problème de la rotation d'un corps solide autour d'un point fixe dans le cas compliqué de Kowalewski et on montre que celui-ci est algébriquement complètement intégrable. On examine ensuite la complète intégrabilité algébrique du flot géodésique sur le groupe $S O(4)$ pour une métrique invariante à gauche. Le problème du mouvement d'un solide dans un fluide parfait, est un cas limite du flot géodésique sur $S O(4)$, ce qui suggère que les cas intégrables de Clebsch et Lyapunov-Steklov soient limites de situations intégrables sur $S O(4)$. Une autre partie, est consacrée à l'étude du système différentiel de Hénon-Heiles. Celui-ci fournit un modèle pour le mouvement d'une étoile dans une galaxie cylindrique ainsi que pour les oscillations des atomes dans une molécule. On montre que la fibre définie par l'intersection des invariants de ce système se complète en une surface lisse et projective, par l'adjonction d'une surface de Riemann $\Gamma$ lisse hyperelliptique de genre trois; laquelle est un revêtement double ramifié le long d'une courbe elliptique. On caractérise cette surface comme étant une variété kählérienne, une variété de Hodge et plus précisément une surface abélienne. Aussi nous prouvons que celle-ci peut être identifiée à la duale d'une variété de Prym et le système en question se linéarise sur cette variété. Ensuite, on passe à l'étude de la complète intégrabilité algébrique d'un potentiel quartique lié au système de Garnier ainsi qu'aux interactions avec les équations couplées non-linéaires de Schrödinger et le champ de Yang-Mills.

\section{Définitions et propriétés}

Considérons le système hamiltonien

$$
\frac{d x(t)}{d t}=J \frac{\partial H}{\partial x}, x \in \mathbb{R}^{m}
$$

avec $H: M \longrightarrow \mathbb{R}$, l'hamiltonien et $J=J(x)$ une matrice antisymétrique satisfaisant à l'identité de Jacobi :

$$
\{\{H, F\}, G\}+\{\{F, G\}, H\}+\{\{G, H\}, F\}=0,
$$


où

$$
\{H, F\}=\left\langle\frac{\partial H}{\partial x}, J \frac{\partial F}{\partial x}\right\rangle=\sum_{i, j} J_{i j} \frac{\partial H}{\partial x_{i}} \frac{\partial F}{\partial x_{j}},
$$

sont les crochets de Poisson.

Definition 2.1. On dit que le système (2.1) est complètement intégrable lorsque :

i) si dét $J \neq 0$, alors $m=2 n$ (le rang d'une matrice antisymétrique est toujours pair). Le système possède $n$ intégrales premières $H_{1}=H, H_{2}, \ldots, H_{n}$, en involution (i.e., $\left\{H_{i}, H_{j}\right\}=0,1 \leq i, j \leq n$ ) et fonctionnellement indépendantes (i.e., $d H_{1} \wedge \ldots \wedge d H_{n} \neq 0$ ). D'après le théorème d'ArnoldLiouville [4, 3, 12], si pour presque tous les $c_{i} \in \mathbb{R}$ les variétés invariantes $\bigcap_{i=1}^{n}\left\{x \in \mathbb{R}^{2 n}: H_{i}(x)=c_{i}\right\}$, sont compactes et connexes, alors elles sont difféomorphes au tore réel $\mathbb{R}^{n} /$ réseau. En outre les flots $g_{X_{i}}^{t}(x)$ définis par les champs de vecteurs $X_{H_{i}}, 1 \leq i \leq n$, sont des mouvements rectilignes. Ces flots déterminent sur ce tore un mouvement quasi-périodique et les équations du problème sont intégrables par quadratures c'est-à-dire les solutions exactes s'expriment par un nombre fini de calculs d'intégrales et d'autres opérations algébriques.

ii) si dét $J=0$, on réduit dans ce cas le problème à $m=2 n+k$ et on cherche $k$ intégrales premières $H_{n+1}, \ldots, H_{n+k}$, dites triviales ou fonctions de Casimir telles que : $J \frac{\partial H_{n+i}}{\partial x}=0,1 \leq i \leq k$. Puis ce qui a été dit dans $\left.i\right)$ s'applique ici pour la variété $\left(\bigcap_{i=1}^{k}\left\{x: H_{n+i}(x)=c_{n+i}\right\}\right) \cap \mathbb{R}^{m}$, de dimension $m-k=2 n$. Si les mêmes conditions sont remplies, alors les variétés $\bigcap_{i=1}^{n+k}\left\{x \in \mathbb{R}^{m}: H_{i}(x)=c_{i}\right\}$, sont difféomorphes au tore réel de dimension $n$.

Supposons que le système différentiel (2.1) soit complètement intégrable. Soit $\Delta \subset \mathbb{C}^{m}$ un ouvert non vide de Zariski, $x \in \mathbb{C}^{m}, t \in \mathbb{C}$ et considérons l'application moment $\varphi:\left(H_{1}, \ldots, H_{n+k}\right): \mathbb{C}^{m} \rightarrow \mathbb{C}^{n+k}$, où les fonctions $H_{1}, \ldots, H_{n+k}$ sont polynomiales. Comme $d H_{1} \wedge \ldots \wedge d H_{n+k} \neq 0$, alors l'application $\varphi$ est une submersion générique sur $\Delta$. Soit $\mathbf{I}=\varphi\left(\mathbb{C}^{m} \backslash \Delta\right)$, i.e.,

$$
\mathbf{I}=\left\{c=\left(c_{i}\right) \in \mathbb{C}^{n+k}: \exists x \in \varphi^{-1}(c) \text { avec } d H_{1}(x) \wedge \ldots \wedge d H_{n+k}(x)=0\right\},
$$

le lieu critique de $\varphi$ où $c=\left(c_{i}\right)$ est le point courant de $\mathbb{C}^{m}$ et désignons par $a d h \mathbf{I}$ l'adhérence (ou fermeture) de Zariski de $\mathbf{I}$ dans $\mathbb{C}^{n+k}$.

Definition 2.2. Le système (2.1) dont le côté droit est polynomial est dit algébriquement complètement intégrable [3, 20] si pour $c \in \mathbb{C}^{n+k} \backslash$ adhI, la 
fibre

$$
M_{c}=\varphi^{-1}(c)=\bigcap_{i=1}^{n+k}\left\{x \in \mathbb{C}^{m}: H_{i}(x)=c_{i}\right\},
$$

est la partie affine d'une variété abélienne (tore complexe algébrique $\widetilde{M}_{c} \simeq$ $\mathbb{C}^{n} /$ réseau). En outre, les flots $g_{X_{i}}^{t}(x), x \in M_{c} t \in \mathbb{C}$, définies par les champs de vecteurs $X_{H_{1}}, \ldots, X_{H_{n}}$ sont des lignes droites sur $\mathbb{C}^{n} /$ réseau c'està-dire $\left[g_{X_{i}}^{t}(x)\right]_{j}=f_{j}\left(p+t\left(k_{1}^{i}, \ldots, k_{n}^{i}\right)\right)$, ò̀ $f_{j}\left(t_{1}, \ldots, t_{n}\right)$ sont des fonctions abéliennes sur $\mathbb{C}^{n} /$ réseau, $f_{j}(p)=x_{j}, 1 \leq j \leq m$.

Notons que $X_{H_{i}}$ définissent sur le tore réel $\mathbb{T}_{c}=\bigcap_{i=1}^{n+k}\left\{x \in \mathbb{R}^{m}: H_{i}(x)=c_{i}\right\}$, une loi d'addition $\oplus: \mathbb{T}_{c} \times \mathbb{T}_{c} \longrightarrow \mathbb{T}_{c},(x, y) \longmapsto x \oplus y=\varphi^{t+s}(p), p \in \mathbb{T}_{c}$, avec $x=\varphi^{t}(p), y=\varphi^{s}(p), \varphi^{t}(p)=\varphi_{X_{1}}^{t_{1}} \ldots \varphi_{X_{n}}^{t_{n}}(p)$, où $\varphi_{X_{i}}^{t_{i}}(p)$ désignent les flots de $X_{H_{i}}$. La complète intégrabilité algébrique du système (2.1) signifie que cette loi d'addition est rationnelle, i.e., $(x \oplus y)_{j}=R_{j}\left(x_{i}, y_{i}, c\right)$ où $R_{j}\left(x_{i}, y_{i}, c\right)$ est une fonction rationnelle des coordonnées $x_{i}, y_{i}$. En posant $x=p, y=\varphi_{X_{i}}^{t}(p)$, dans cette formule, on remarque que sur le tore réel $\mathbb{T}_{c}$, les flots $\varphi_{i}^{t}(p)$ dépendent rationnellement de la condition initiale $p$. Par ailleurs, un théorème de Weierstrass sur les fonctions admettant un théorème d'addition, affirme que les coordonnées $x_{i}$ restreintes au tore réel : $\mathbb{R}^{n} /$ réseau $\longrightarrow \mathbb{T}_{c},\left(t_{1}, \ldots, t_{n}\right) \longmapsto x_{i}\left(t_{1}, \ldots, t_{n}\right)$, sont des fonctions abéliennes. Géométriquement, cela signifie que le tore réel $\mathbb{T}_{c} \simeq \mathbb{R}^{n} /$ réseau est la partie affine d'un tore complexe algébrique $\mathbb{C}^{n} /$ réseau (variété abélienne) et que les fonctions réelles $x_{i}\left(t_{1}, \ldots, t_{n}\right),\left(t_{i} \in \mathbb{R}\right)$, sont les restrictions sur ce tore réel des fonctions méromorphes $x_{i}\left(t_{1}, \ldots, t_{n}\right),\left(t_{i} \in \mathbb{C}\right)$, de $n$ variables complexes. La définition 2.2 signifie qu'on peut trouver une transformation algébrique $\left(x_{1}(t), \ldots, x_{n}(t)\right) \mapsto\left(s_{1}(t), \ldots, s_{n}(t)\right)$, telle que : $\sum_{i=1}^{n} \int_{s_{i}(0)}^{s_{i}(t)} \omega_{k}=a_{k} t, k=1, \ldots, n$ où $\omega_{1}, \ldots, \omega_{n}$ sont des différentielles holomorphes sur une courbe algébrique liée au problème. Comme le flot évolue sur le tore complexe $\widetilde{M}_{c} \simeq \mathbb{C}^{n} /$ réseau, les coordonnées $x_{i}$ restent finies sur la partie affine (non compacte) $M_{c}$ de ce tore et en outre les coordonnées $x_{1}, \ldots, x_{m}$ doivent tendre vers l'infini le long d'un diviseur $\mathcal{D} \subset \mathbb{C}^{n} /$ réseau. Par la définition de la complète intégrabilité algébrique, le flot (2.1) est une ligne droite (un mouvement rectiligne) sur $\mathbb{C}^{n} /$ réseau, chacune de ces trajectoires doit cependant heurter la sous-variété $\mathcal{D}$ en au moins un endroit. Réciproquement, à travers tout point de $\mathcal{D}$ il existe un mouvement rectiligne et par conséquent un développement de Laurent autour de ce point d'intersection. D'où les équations différentielles (2.1) doivent admettre des séries de Laurent lesquelles dépendent des $n-1$ paramètres définissant $\mathcal{D}$ et des $n+k$ constantes $c_{i}$ définissant 
$\mathcal{D}^{n} /$ réseau c'est-à-dire les séries autour des points d'intersection, dépendent de $(n+k)+(n-1)=m-1=\operatorname{dim}$ (espace de phase) -1 paramètres libres.

\section{Construction des variétés abéliennes et linéarisation}

En vertu du théorème de Noether [4], les groupes à un paramètre de difféomorphismes d'un système dynamique définissent les intégrales premières. Les variétés de niveau communes de ces intégrales premières sont des variétés invariantes du flot. La solution d'un problème non-linéaire se ramène actuellement, à l'étude topologique de son flot et de ces variétés invariantes. Les méthodes de résolution sont étroitement liée à un arsenal très riche de spécialités mathématiques : théorie spectrale, groupes et algèbres de Lie, géométrie différentielle, géométrie algébrique et plus particuliérement les surfaces de Riemann ainsi que les variétés abéliennes qui ont eu là une très heureuse influence. Les solutions de ces problèmes ont toujours été exprimés en termes d'intégrales hyperelliptiques. Autrement dit, le problème semble se linéariser sur la variété jacobienne d'une surface de Riemann hyperelliptique. Or plusieurs études récentes $[1,2,3,6,9,10,14]$ ont montré que les tores invariants, vus dans le complexe, ne sont pas principalement polarisés, mais sont au mieux isogènes à des surfaces principalement polarisées. Actuellement on sait qu'en genre 2, principalement polarisé équivaut à hyperelliptique, donc toute surface abélienne est isogène à une jacobienne hyperelliptique. Ceci explique pourquoi les anciens ont toujours été capables de trouver des solutions en termes d'intégrales hyperelliptiques. Dans chacun des cas la méthode de résolution est compliquée et repose sur un choix astucieux de variables.

Pour aider à comprendre ces difficultés, prenons le cas d'un système nonlinéaire dans $\mathbb{C}^{6}$, dont les solutions s'expriment en termes de fonctions hyperelliptiques de genre 2 . Soit $M$ la variété invariante du flot correspondant à ce système et considérons le diagramme suivant :

$$
\begin{array}{ccc}
M \subset \widetilde{M} & = & \operatorname{Jac}(\mathcal{H}) \\
(i) \downarrow & & \searrow(i i i) \\
J a c\left\{s_{1}+s_{2}\right\} / \equiv & \underset{(i i)}{\simeq} & \mathbb{C}^{2} / L_{\Omega}
\end{array}
$$

où $\mathcal{H}$ est une surface de Riemann hyperelliptique de genre 2 , d'équation affine $w^{2}=P(z)$ avec $P(z)$ un polynôme de degré cinq ou six. Ici $s_{1}$ et $s_{2}$ désignent les fameuses variables qu'il faut trouver pour pouvoir résoudre le problème. Le chemin $(i)$ et $(i i)$ correspond à ce qu'on appelle résolution ou intégration 
du problème par quadrature. Il s'agit de trouver la transformation

$$
\begin{gathered}
\operatorname{Jac}\left\{s_{1}+s_{2}\right\} / \equiv \stackrel{\simeq}{\longrightarrow} \mathbb{C}^{2} / L_{\Omega}, \\
s_{1}+s_{2} \mapsto \int^{s_{1}(t)}\left(\omega_{1}, \omega_{2}\right)+\int^{s_{2}(t)}\left(\omega_{1}, \omega_{2}\right)=t\left(k_{1}, k_{2}\right), \quad\left(k_{1}, k_{2} \in \mathbb{C}\right),
\end{gathered}
$$

où $\omega_{1}=\frac{d z}{\sqrt{P(z)}}, \omega_{2}=\frac{z d z}{\sqrt{P(z)}}$, sont des différentielles holomorphes sur la surface de Riemann $\mathcal{H}$ et

$$
\frac{d s_{1}}{\sqrt{P\left(s_{1}\right)}}+\frac{d s_{2}}{\sqrt{P\left(s_{2}\right)}}=k_{1} d t, \quad \frac{s_{1} d s_{1}}{\sqrt{P\left(s_{1}\right)}}+\frac{s_{2} d s_{2}}{\sqrt{P\left(s_{2}\right)}}=k_{2} d t .
$$

En pratique il est difficile de trouver les variables $s_{1}$ et $s_{2}$. En fait, la découverte de l'intégrabilité d'un système était souvent due à un coup de chance, sans toutefois en comprendre les raisons ou les mécanismes. La stratégie de résolution que nous avons évoqué dans l'introduction vise une approche systématique et directe pour résoudre ces problèmes non-linéaires. Elle correspond au chemin (iii) (version géométrique du théorème d'Arnold-Liouville) : $M \subset \widetilde{M}=\operatorname{Jac}(\mathcal{H}) \longrightarrow \mathbb{C}^{2} / L_{\Omega}, g_{X_{1}}^{t_{1}} \circ g_{X_{2}}^{t_{2}}(p) \curvearrowleft\left(t_{1}, t_{2}\right)$, mais il faut que la variété $M$ soit compacte, sinon il faut la compactifier.

Si le système de ces équations est algébriquement intégrable, alors il possède une famille de solutions en séries de Laurent dépendant d'un nombre suffisant de paramètres libres. Ces solutions évoluent selon des mouvements rectilignes en temps complexe sur des variétés abéliennes (tores complexes algébriques). Ces variétés ont toute une série de propriétés remarquables. Par exemple, le théorème de Kodaira [5, 15] combiné avec le théorème de Lefschetz $[5,15]$ affirme que les fonctions méromorphes sur le tore ayant un pôle d'ordre un, deux ou trois le long d'un diviseur positif, permettent de plonger le tore de façon lisse dans un espace projectif. Une étape cruciale consiste à trouver ce diviseur positif; il s'avère que celui-ci est défini par les relations entre les paramètres libres obtenus en confinant les solutions en séries de Laurent aux variétés invariantes. A partir d'un diviseur sur une variété abélienne, on peut effectivement avoir un grand nombre de renseignements sur les tores, ce qui finalement, permet d'écrire les intégrales abéliennes et donc linéariser les équations différentielles. En utilisant la formule d'adjonction [5, 15], on montre le résultat suivant:

Theorem 3.1. Soit $\bar{M}_{c}$ la fermeture projective de $M_{c}(2.2)$ dans l'espace projectif $\mathbb{C P}^{m}$ de dimension $m$. Alors $\bar{M}_{c}$ n'est pas une variété abélienne et elle est singulière à l'infini. 
Démonstration : Soient $Z_{0}, \ldots, Z_{m}$ des coordonnées homogènes dans l'espace projectif complexe $\mathbb{C P}^{m}$ et $\overline{M_{c}}$ la fermeture projective de $M_{c}$ dans $\mathbb{C P}^{m}$. On va utiliser un raisonnement par l'absurde. Supposons que $\overline{M_{c}}$ soit une variété abélienne considérée comme étant le quotient $\mathbb{C}^{n} / L$ de $\mathbb{C}^{n}$ par un réseau $L$ isomorphe à $\mathbb{Z}^{n}$. Donc $\overline{M_{c}}$ est lisse et isomorphe à une sous-variété fermée sans singularité et dès lors toute forme différentielle de la forme $d \tau_{1} \wedge \ldots \wedge d \tau_{n}$ $\left(\tau_{1}, \ldots, \tau_{n}\right.$ sont des coordonnées naturelles sur $\left.\overline{M_{c}} \simeq \mathbb{C}^{n} / L\right)$ est évidemment holomorphe et ne s'annule jamais. Pour aboutir à une contradiction, on va calculer le fibré canonique $K_{\overline{M_{c}}}$ de $\overline{M_{c}}$, à l'aide de la formule d'adjonction $[5]: K_{\overline{M_{c}}}=\left.\left(K_{\mathbb{C P}^{m}} \otimes\left[\overline{M_{c}}\right]\right)\right|_{\overline{M_{c}}}$ où $\left[\overline{M_{c}}\right.$ est le fibré en droites dans $\mathbb{C P}^{m}$. Le fibré canonique $K_{\mathbb{C P}^{m}}$ de $\mathbb{C P}^{m}$ est $K_{\mathbb{C P}^{m}}=[(\omega)]=\left[-(m+1) \mathbf{H}_{\infty}\right]$ où $\mathbf{H}_{\infty}$ est un hyperplan dans $\mathbb{C P}^{m}$. En remplaçant dans la formule d'adjonction précédente, on obtient $\left.K_{\overline{M_{c}}} \sim\left[\mathbf{H}_{\infty}\right]\right|_{\overline{M_{c}}}$ et donc toute forme différentielle sur $\overline{M_{c}}$ doit avoir un zéro sur $\overline{M_{c}}$, ce qui est contradictoire.

D'après ce théorème, pour que $M_{c}$ soit la partie affine d'une variété abélienne, la variété $\bar{M}_{c}$ doit être singulière à l'infini c'est-à-dire le long du lieu $Z_{0}=0$. Soit $\mathcal{D}$ le diviseur $\bar{M}_{c} \cap \mathbf{H}_{\infty}$ où $\mathbf{H}_{\infty}$ est l'hyperplan à l'infini défini par $Z_{0}=0$. En éclatant la singularité le long du lieu atteint par le flot et en implosant la partie du lieu qui n'est pas atteint par le flot, on montre que la variété $\bar{M}_{c}$ se transforme en une variété abélienne $\widetilde{M}_{c}$ et le lieu à l'infini $\mathcal{D}$ se transforme en une ou plusieurs sous-variétés de codimension 1 . On procède comme suit : soit $x_{i} \longrightarrow y_{i}$ une transformation birationnelle telle qu'au voisinage de $t=0$, on ait : $y_{i}=\alpha_{i}+o(t)$ pour $1 \leq i \leq m-1$, et $y_{m}=t+o\left(t^{2}\right)$, où $\alpha_{1}, \ldots, \alpha_{m-1}$ sont des paramètres libres. Les nouvelles variables $y_{i}$ ont pour effet d'éclater la singularité de la variété projective $\bar{M}_{c}$ le long du lieu $Z_{0}=0$ atteint par le flot. Autrement dit, les équations différentielles suggèrent d'éclater la singularité en séparant les feuillets le long du lieu dans $Z_{0}=0$ atteint par le flot et suggèrent d'imploser la partie du lieu qui n'est pas atteint par le flot. Exprimées dans ces nouvelles variables $y_{1}, \ldots, y_{m}$, les équations différentielles sont régulières et holomorphes au voisinage de $y_{m}=0$ tandis que les équations définissant la fibre $M_{c}$ s'écrivent sous la forme : $F_{i}\left(y_{1}(t), \ldots, y_{m-1}(t), y_{m}(t)\right)=c_{i}$, $1 \leq i \leq n+k$, où $F_{1}, \ldots, F_{n+k}$ sont des polynômes en $y$. Pour $t=0$, on obtient les relations algébriques : $F_{i}\left(\alpha_{1}, \ldots, \alpha_{m-1}, 0\right)=c_{i}, 1 \leq i \leq n+k$, entre les paramètres libres $\alpha_{1}, \ldots, \alpha_{m-1}$. Ces relations fournissent les équations de la sous-variété $\mathcal{D}$. En fait, les paramètres libres $\alpha_{1}, \ldots, \alpha_{m-1}$ et la sous variété $\mathcal{D}$ peuvent s'obtenir directement de la manière suivante : d'abord l'on montre l'existence de solutions $x=\left(x_{1}, x_{2}, \ldots, x_{m}\right)$ du système (2.1) sous la forme de séries de Laurent $x_{i}=\sum_{k=0}^{\infty} x_{i}^{(k)} t^{k-s_{i}}, 1 \leq i \leq m$, dépendant de $m-1$ paramètres libres $\alpha_{1}, \ldots, \alpha_{m-1}$. Ici $s_{1}, \ldots, s_{m}$ sont des entiers positifs. En sub- 
stituant ces développements dans le système (2.1), on voit que les coefficients $x^{(0)}, x^{(1)}, \ldots$, satisfont aux équations

$$
\begin{gathered}
s_{i} x_{i}^{(0)}+f_{i}\left(x_{1}^{(0)}, \ldots, x_{m}^{(0)}\right)=0, \\
(L-k I) x^{(k)}=\left\{\begin{aligned}
0 & \text { pour } k=1, \\
\text { polynôme en } x_{i}^{(0)}, \ldots, x_{i}^{(k-1)} & \text { pour } k \geq 2,
\end{aligned}\right.
\end{gathered}
$$

où $L$ est la matrice jacobienne de (3.1). Des paramètres libres apparaissent dans le système d'équations non-linéaires (3.1) lorsque celui-ci admet un ensemble continu de solutions et dans le système d'équations linéaires (3.2) du fait que $k \in \mathbb{N}^{*}$ est une valeur propre de la matrice $L$. L'étape suivante est fondamentale et consiste à considérer l'ensemble :

$$
\mathcal{D}=\bigcap_{i=1}^{n+k}\left\{\text { coefficient de } t^{0} \text { dans } H_{i}(x(t))=c_{i}\right\}
$$

Ensuite on procède à la compactification de la fibre $M_{c}(2.2)$ en une variété abélienne $\widetilde{M}_{c}$.

Theorem 3.2. Soient $\mathcal{S} \equiv k \mathcal{D}$ où $k=1,2,3$ et $V_{p}$ une variété lisse telle que $: V_{p}=\widetilde{M}_{c}, V_{p} \backslash \mathcal{S} \subseteq M_{c}$ dans un voisinage de $p$. Alors, la variété $\widetilde{M}_{c}=M_{c} \cup\left(\bigcup_{p \in \mathcal{S}} V_{p}\right)$, est compacte, connexe et possède un plongement dans l'espace projectif complexe $\mathbb{C P}^{N}$. En particulier $\mathcal{S}$ est très ample.

Démonstration : Soit $p \in \mathcal{S}, \varepsilon>0$ suffisamment petit, $g_{X}^{t}$ le flot correspondant au champ de vecteurs $X_{H}(2.1)$ et $\left\{g_{X}^{t}: t \in \mathbb{C}, 0<|t|<\varepsilon\right\}$ l'orbite passant à travers le point $p$. Posons $V \equiv \bigcup_{p \in \mathcal{S}} V_{p}$ où $V_{p}$ est l'élément dans $\mathbb{C P}^{N}$ formé par le diviseur $\mathcal{S}$ et les orbites ci-dessus et considérons la variété $V^{\prime}=\mathcal{H} \cap V$ où $\mathcal{H} \subset \mathbb{C P}^{N}$ est un hyperplan transverse à la direction du flot. Notons que $V^{\prime}$ est lisse (en effet, supposons que $V^{\prime}$ est singulière en 0 ce qui implique que $V$ est singulière le long de la trajectoire (axe des $t$ ) laquelle pénètre immédiatement dans la partie affine $M_{c}$ donc celle-ci est singulière ce qui est absurde car la fibre $M_{c}$ est une variété affine lisse (fibré d'un morphisme de $\mathbb{C}^{m}$ dans $\left.\mathbb{C}^{n+k}\right)$ ) et d'après le théorème des fonctions implicites la variété $V$ est aussi lisse. Considérons l'application $\bar{M}_{c} \subset \mathbb{C P}^{m} \rightarrow \mathbb{C P}^{N}, X \mapsto f(X)$, où $X=\left(X_{0}, X_{1}, \ldots, X_{m}\right)$ et $\widetilde{M}_{c}=f\left(\bar{M}_{c}\right)$. Rappelons que $\bar{M}_{c}$ est la fermeture projective de $M_{c}$ dans $\mathbb{C P}^{m}$ et $f=\left(1, f_{1}, \ldots, f_{N}\right)$, est une base de l'espace $\mathcal{L}(\mathcal{S})$. Dans un voisinage $\left(\subset \mathbb{C P}^{N}\right)$ de $p$, on a $V_{p}=\widetilde{M}_{c}$ et $V_{p} \backslash \mathcal{S} \subset M_{c}$ car sinon, il existe un élément $V_{p}^{\prime} \subset \widetilde{M}_{c}$ tel que : $V_{p} \cap V_{p}^{\prime}=$ axe des $t$ et orbite $\left\{g_{X}^{t}: t \in \mathbb{C}, 0<|t|<\varepsilon\right\}=($ axe des $t) \backslash p \subset M_{c}$ et $M_{c}$ serait singulière 
le long des axes des $t$, ce qui est impossible d'après ce qui précède. La variété $\bar{M}_{c} \cap\left\{X_{0} \neq 0\right\}$, étant irréductible et comme la section (générique) hyperplane $\mathcal{H}_{\text {gen. }}$ de $\bar{M}_{c}$ est aussi irréductible, alors toutes les sections hyperplanes sont connexes et par conséquent le lieu à l'infini $I=\bar{M}_{c} \cap \mathbf{H}_{\infty}$, (où $\mathbf{H}_{\infty}$ est l'hyperplan à l'infini défini par $\left.X_{0}=0\right)$ est aussi connexe. Soit $\Gamma_{f} \subset \mathbb{C P}^{m} \times \mathbb{C P}^{N}$, le graphe de l'application $f$, lequel est irréductible ensemble avec $\bar{M}_{c}$. Il s'ensuit de l'irréductibilité de $I$ qu'une section (générique) hyperplane est irréductible, d'où la section (spéciale) hyperplane $\Gamma_{f} \cap\left(\mathbb{C P}^{N} \times\left\{X_{0}=0\right\}\right)$ est connexe et donc l'application projective $\operatorname{Proj}_{\mathbb{C P}^{N}}\left[\Gamma_{f} \cap\left(\mathbb{C P}^{N} \times\left\{X_{0}=0\right\}\right)\right]=f(I)=\mathcal{S}$ est connexe. La variété $\widetilde{M}_{c}=M_{c} \cup V$ est compacte, connexe et possède un plongement dans l'espace projectif complexe $\mathbb{C P}^{N}$ via l'application $f$.

Remark 3.1. On montre qu'il existe sur la variété $\widetilde{M}_{c} n$ - différentielles holomorphes $d t_{1}, \ldots, d t_{n}$ telles que : $\left.d t_{1}\right|_{\mathcal{D}}=\omega_{1}, \ldots,\left.d t_{n}\right|_{\mathcal{D}}=\omega_{n}$ où $\omega_{1}, \ldots, \omega_{n}$ sont des différentielles holomorphes sur $\mathcal{D}$. L'espace des différentielles holomorphes sur $\mathcal{S}$ est $\left\{f_{i}^{(0)} \omega_{1}, 1 \leq i \leq N\right\} \oplus\left\{\omega_{1}, \ldots, \omega_{n}\right\}$, où les $f_{i}^{(0)}$ sont les premiers coefficients des fonctions $f_{i} \in \mathcal{L}(\mathcal{S})$ et le plongement de $\mathcal{D}$ dans $\mathbb{C P}^{N}$ est à $n$ différentielles holomorphes près le plongement canonique : $\left(\alpha_{1}, \ldots, \alpha_{n}\right) \in \mathcal{D} \longmapsto\left[\omega_{1}, f_{1}^{(0)} \omega_{1}, \ldots, f_{N}^{(0)} \omega_{1}\right] \in \mathbb{C P}^{N}$.

Rappelons que le théorème d'Arnold-Liouville [4, 12] joue un rôle crucial dans l'étude des systèmes intégrables. Il permet, entre autres, d'étudier la situation topologique suivante : si les variétés invariantes sont compactes et connexes, alors elles sont difféomorphes aux tores réels sur lequels le flot de phase détermine un mouvement quasi-périodique. Les équations du problème à étudier sont intégrables par quadratures et le théorème en question montre un comportement très régulier des solutions. La preuve que nous allons donner ici s'inspire de celle du théorème d'Arnold-Liouville dans le cas réel.

Theorem 3.3. La variété $\widetilde{M}_{c}$ est un tore complexe $\widetilde{M}_{c} \simeq \mathbb{C}^{n} / L$ où L est un réseau de $\mathbb{C}^{n}$. En particulier, $\widetilde{M}_{c}$ est une variété holomorphe kählérienne.

Démonstration : Soient $g_{X_{1}}^{t_{1}}, \ldots, g_{X_{n}}^{t_{2}}$ les flots engendrés respectivement par les champs de vecteurs $X_{H_{1}}, \ldots, X_{H_{n}}$ et $p_{1} \in \widetilde{M}_{c} \backslash M_{c}$. Pour $\varepsilon>0$ suffisamment petit, $g_{X_{1}}^{t_{1}}\left(p_{1}\right), \forall t_{1} \in \mathbb{C}, 0<\left|t_{1}\right|<\varepsilon$, est bien définie et $g_{X_{1}}^{t_{1}}\left(p_{1}\right) \in M_{c}$. Soit $\mathcal{V}(q) \subset M_{c}$ un voisinage de $q \equiv g_{X_{1}}^{t_{1}}\left(p_{1}\right)$ et posons $g_{X_{2}}^{t_{2}}\left(p_{2}\right)=g_{X_{1}}^{-t_{1}} \circ g_{X_{2}}^{t_{2}} \circ$ $g_{X_{1}}^{t_{1}}\left(p_{2}\right), \forall p_{2} \in \mathcal{V}\left(p_{1}\right) \equiv g^{-t_{1}}(\mathcal{V}(q))$. Cette définition a bien un sens car $g_{X_{2}}^{t_{2}}$ est indépendant de $t_{2}$ puisque $g_{X_{1}}^{-\left(t_{1}+\varepsilon\right)} \circ g_{X_{2}}^{t_{2}} \circ g_{X_{1}}^{t_{1}+\varepsilon}\left(p_{2}\right)=g_{X_{1}}^{-t_{1}} \circ g_{X_{2}}^{t_{2}} \circ g_{X_{1}}^{t_{1}}\left(p_{2}\right)$ en vertu de la commutativité des champs de vecteurs. La fonction $g_{X_{2}}^{t_{2}}\left(p_{2}\right)$ est holomorphe en $p_{2}$ et $t_{2}$. Il en est de même pour $g_{X_{3}}^{t_{3}}\left(p_{3}\right), \ldots, g_{X_{n}}^{t_{n}}\left(p_{n}\right)$ avec $g_{X_{n}}^{t_{n}}\left(p_{n}\right)=g_{X_{n}}^{-t_{n-1}} \circ g_{X_{n}}^{t_{n}} \circ g_{X_{n}}^{t_{n-1}}\left(p_{n}\right), \forall p_{n} \in \mathcal{V}\left(p_{n-1}\right) \equiv g^{-t_{n-1}}(\mathcal{V}(q))$. 
Les champs de vecteurs $X_{H_{1}}, \ldots, X_{H_{n}}$ se prolongent de façon holomorphe et demeurent indépendants sur la variété $\widetilde{M}_{c}$. Fixons maintenant $p \in \widetilde{M}_{c}$ et considérons $\mathbb{C}^{n} \rightarrow \widetilde{M}_{c},\left(t_{1}, \ldots, t_{n}\right) \mapsto g\left(t_{1}, \ldots, t_{n}\right)=g^{t_{1}} \circ \ldots \circ g^{t_{n}}(p)$ et $L=\left\{\left(t_{1}, \ldots, t_{n}\right) \in \mathbb{C}^{n}: g\left(t_{1}, \ldots, t_{n}\right)=p\right\}$. Comme $\widetilde{M}_{c}$ est compact, on montre que $L$ est un réseau de $\mathbb{C}^{n}$ engendré par $2 n$ vecteurs (dans $\left.\mathbb{C}^{n}\right) \mathbb{R}$-linéairement indépendants. En faisant le quotient de $\mathbb{C}^{n}$ par $L$, on montre que $\widetilde{M}_{c}$ est difféomorphe au tore complexe $\mathbb{C}^{n} / L$. Munissons la variété complexe $\widetilde{M}_{c}$ de la métrique hermitienne $d s^{2}=\sum_{k=1}^{n} d t_{k} \otimes d \overline{t_{k}}$ et soit $\omega=-\frac{1}{2} \operatorname{Im} d s^{2}$ la $(1,1)$-forme fondamentale associée à cette métrique ; i.e., $\omega=\frac{\sqrt{-1}}{2} \sum_{k=1}^{n} d t_{k} \wedge d \overline{t_{k}}$. Comme $\omega$ est fermée, on en déduit que $d s^{2}$ est une métrique kählérienne et que la variété $\widetilde{M}_{c}$ est kählérienne.

Remark 3.2. Nous avons démontré dans le théorème précédent, que la variété complexe $\widetilde{M}_{c}$ est kählérienne. En utilisant le critère de Moishezon [18], on peut donner une nouvelle preuve que $\widetilde{M}_{c}$ est projective.

En outre, on montre que :

Theorem 3.4. La variété $\widetilde{M}_{c}$ est de Hodge. En outre, la fibre $M_{c}(2.2)$ forme la partie affine d'une variété abélienne $\widetilde{M}_{c}$ et le système (2.1) est algébriquement complètement intégrable.

Démonstration : Les notations sont comme dans la démonstration du théorème précédent. Les intégrales $\int_{\gamma_{k}} \omega$ de la forme $\omega$ où $\gamma_{k}$ sont des cycles à deux dimensions dans $H_{2}\left(\widetilde{M}_{c}, \mathbb{Z}\right)$, déterminent les périodes de $\omega$. Comme celles-ci sont des nombres entiers, alors la variété $\widetilde{M}_{c}$ est de Hodge. La variété $\widetilde{M}_{c}$ est munie de $n$ champs de vecteurs réguliers, indépendants en chaque point et commutants. D'après le théorème 3.3 , la variété $\widetilde{M}_{c}$ est un tore complexe et comme celui-ci possède un plongement projectif (théorème 3.2 ou remarque 3.1 , alors $\widetilde{M}_{c}$ est une variété abélienne. Une autre preuve consiste à utiliser le fait que $\widetilde{M}_{c}$ est de Hodge puisque tout tore de Hodge est abélien. La réciproque est aussi vraie.

\section{Applications}

Cette section est consacrée à l'étude de quelques exemples importants de systèmes dynamiques algébriquement complètement intégrables. 


\subsection{La toupie de Kowalewski}

Les équations du mouvement d'un corps solide autour d'un point fixe s'écrivent sous la forme

$$
\begin{aligned}
\frac{d M}{d t} & =M \wedge \Omega+\mu g \Gamma \wedge L, \\
\frac{d \Gamma}{d t} & =\Gamma \wedge \Omega
\end{aligned}
$$

où $\wedge$ est le produit vectoriel dans $\mathbb{R}^{3}, M=\left(m_{1}, m_{2}, m_{3}\right)$ le moment angulaire $\mathrm{du}$ solide, $\Omega=\left(m_{1} / I_{1}, m_{2} / I_{2}, m_{3} / I_{3}\right)$ la vitesse angulaire, $I_{1}, I_{2}$ et $I_{3}$, les moments d'inertie, $\Gamma=\left(\gamma_{1}, \gamma_{2}, \gamma_{3}\right)$ le vecteur vertical unitaire, $\mu$ la masse du solide, $g$ l'accélération de la pesanteur, et enfin, $L=\left(l_{1}, l_{2}, l_{3}\right)$ le vecteur unitaire ayant pour origine le point fixe et dirigé vers le centre de gravité ; tous ces vecteurs sont considérés dans un système mobile dont les coordonnées sont fixées aux axes principaux d'inertie. L'espace de configuration d'un solide avec un point fixe est le groupe des rotations $S O(3)$. C'est le groupe des matrices orthogonales d'ordre trois et le mouvement de ce solide est décrit par une courbe sur ce groupe. L'espace des vitesses angulaires de toutes les rotations est l'algèbre de Lie du groupe $S O(3)$; c'est l'algèbre so(3) des matrices antisymétriques d'ordre trois. Cette algèbre est engendrée comme espace vectoriel par les matrices

$$
e_{1}=\left(\begin{array}{ccc}
0 & 0 & 0 \\
0 & 0 & -1 \\
0 & 1 & 0
\end{array}\right), \quad e_{2}=\left(\begin{array}{ccc}
0 & 0 & 1 \\
0 & 0 & 0 \\
-1 & 0 & 0
\end{array}\right), \quad e_{3}=\left(\begin{array}{ccc}
0 & -1 & 0 \\
1 & 0 & 0 \\
0 & 0 & 0
\end{array}\right),
$$

qui vérifient les relations de commutation $\left[e_{1}, e_{2}\right]=e_{3},\left[e_{2}, e_{3}\right]=e_{1},\left[e_{3}, e_{1}\right]=$ $e_{2}$. On utilisera dans la suite le fait que si l'on identifie so (3) à $\mathbb{R}^{3}$ en envoyant $\left(e_{1}, e_{2}, e_{3}\right)$ sur la base canonique de $\mathbb{R}^{3}$, le crochet de so(3) correspond au produit vectoriel. En d'autres termes, considérons l'application

$$
\mathbb{R}^{3} \longrightarrow s o(3), a=\left(a_{1}, a_{2}, a_{3}\right) \longmapsto A=\left(\begin{array}{ccc}
0 & -a_{3} & a_{2} \\
a_{3} & 0 & -a_{1} \\
-a_{2} & a_{1} & 0
\end{array}\right),
$$

laquelle définit un isomorphisme entre les algèbres de Lie $\left(\mathbb{R}^{3}, \wedge\right)$ et $(s o(3),[]$, où $a \wedge b \longmapsto[A, B]=A B-B A$. En utilisant cet isomorphisme, on peut réecrire le système (4.1) sous la forme

$$
\begin{aligned}
\frac{d M}{d t} & =[M, \Omega]+\mu g[\Gamma, L], \\
\frac{d \Gamma}{d t} & =[\Gamma, \Omega],
\end{aligned}
$$


où

$$
\begin{aligned}
M & =\left(M_{i j}\right)_{1 \leq i, j \leq 3} \equiv \sum_{i=1}^{3} m_{i} e_{i} \equiv\left(\begin{array}{ccc}
0 & -m_{3} & m_{2} \\
m_{3} & 0 & -m_{1} \\
-m_{2} & m_{1} & 0
\end{array}\right) \in s o(3), \\
\Omega & =\left(\Omega_{i j}\right)_{1 \leq i, j \leq 3} \equiv \sum_{i=1}^{3} \omega_{i} e_{i} \equiv\left(\begin{array}{ccc}
0 & -\omega_{3} & \omega_{2} \\
\omega_{3} & 0 & -\omega_{1} \\
-\omega_{2} & \omega_{1} & 0
\end{array}\right) \in s o(3), \\
\Gamma & =\left(\Gamma_{i j}\right)_{1 \leq i, j \leq 3} \equiv \sum_{i=1}^{3} \gamma_{i} e_{i} \equiv\left(\begin{array}{ccc}
0 & -\gamma_{3} & \gamma_{2} \\
\gamma_{3} & 0 & -\gamma_{1} \\
-\gamma_{2} & \gamma_{1} & 0
\end{array}\right) \in s o(3),
\end{aligned}
$$

et

$$
L=\left(\begin{array}{ccc}
0 & -l_{3} & l_{2} \\
l_{3} & 0 & -l_{1} \\
-l_{2} & l_{1} & 0
\end{array}\right)
$$

En tenant compte du fait que $M=I \Omega$, alors les équations pécédentes deviennent

$$
\begin{aligned}
\frac{d M}{d t} & =[M, \Lambda M]+\mu g[\Gamma, L] \\
\frac{d \Gamma}{d t} & =[\Gamma, \Lambda M]
\end{aligned}
$$

où

$$
\Lambda M=\sum_{i=1}^{3} \lambda_{i} m_{i} e_{i} \equiv\left(\begin{array}{ccc}
0 & -\lambda_{3} m_{3} & \lambda_{2} m_{2} \\
\lambda_{3} m_{3} & 0 & -\lambda_{1} m_{1} \\
-\lambda_{2} m_{2} & \lambda_{1} m_{1} & 0
\end{array}\right), \lambda_{i} \equiv I_{i}^{-1}
$$

On sait que le système (4.1) ou (4.2) est complètement intégrable dans les cas suivants : $i$ ) cas d'Euler : $\left.l_{1}=l_{2}=l_{3}=0, i i\right)$ cas de Lagrange : $I_{1}=I_{2}$, $l_{1}=l_{2}=0$ et $\left.i i i\right)$ cas de Kowalewski : $I_{1}=I_{2}=2 I_{3}, l_{3}=0$. L'étude des deux premiers cas ne pose aucun problème, par contre celle du dernier cas est compliquée. Le système différentiel (4.2) s'écrit dans le cas de Kowalewski explicitement sous la forme

$$
\begin{aligned}
\frac{d m_{1}}{d t} & =m_{2} m_{3}, & & \frac{d \gamma_{1}}{d t}=2 m_{3} \gamma_{2}-m_{2} \gamma_{3}, \\
\frac{d m_{2}}{d t} & =-m_{1} m_{3}+2 \gamma_{3}, & \frac{d \gamma_{2}}{d t} & =m_{1} \gamma_{3}-2 m_{3} \gamma_{1}, \\
\frac{d m_{3}}{d t} & =-2 \gamma_{2}, & \frac{d \gamma_{3}}{d t} & =m_{2} \gamma_{1}-m_{1} \gamma_{2},
\end{aligned}
$$


où, sans restreindre la généralité, nous avons choisi $l_{2}=0, \mu g l_{1}=1, I_{3}=1$ et nous avons utilisé la substitution $t \rightarrow 2 t$. Ces équations s'écrivent sous forme d'un champ de vecteurs hamiltonien

$$
\frac{d x}{d t}=J \frac{\partial H}{\partial x}, x=\left(m_{1}, m_{2}, m_{3}, \gamma_{1}, \gamma_{2}, \gamma_{3}\right)^{\top},
$$

avec $H=\frac{1}{2}\left(m_{1}^{2}+m_{2}^{2}\right)+m_{3}^{2}+2 \gamma_{1}$, l'hamiltonien et

$$
J=\left(\begin{array}{cccccc}
0 & -m_{3} & m_{2} & 0 & -\gamma_{3} & \gamma_{2} \\
m_{3} & 0 & -m_{1} & \gamma_{3} & 0 & -\gamma_{1} \\
-m_{2} & m_{1} & 0 & -\gamma_{2} & \gamma_{1} & 0 \\
0 & -\gamma_{3} & \gamma_{2} & 0 & 0 & 0 \\
\gamma_{3} & 0 & -\gamma_{1} & 0 & 0 & 0 \\
-\gamma 2 & \gamma_{1} & 0 & 0 & 0 & 0
\end{array}\right)
$$

Ce système différentiel, admet quatre intégrales premières :

$$
\begin{aligned}
& H_{1} \equiv H \\
& H_{2}=m_{1} \gamma_{1}+m_{2} \gamma_{2}+m_{3} \gamma_{3}, \\
& H_{3}=\gamma_{1}^{2}+\gamma_{2}^{2}+\gamma_{3}^{2} \\
& H_{4}=\left(\left(\frac{m_{1}+i m_{2}}{2}\right)^{2}-\left(\gamma_{1}+i \gamma_{2}\right)\right)\left(\left(\frac{m_{1}-i m_{2}}{2}\right)^{2}-\left(\gamma_{1}-i \gamma_{2}\right)\right) .
\end{aligned}
$$

Le second champ de vecteurs

$$
\frac{d x}{d t}=J \frac{\partial H_{4}}{\partial x}, x=\left(m_{1}, m_{2}, m_{3}, \gamma_{1}, \gamma_{2}, \gamma_{3}\right)^{\top},
$$

est quartique. En outre, les intégrales premières $H_{1}$ et $H_{4}$ sont en involution, tandis que $H_{1}$ et $H_{3}$ sont triviaux. Soit

$$
M_{c}=\bigcap_{k=1}^{4}\left\{x \in \mathbb{C}^{6}: H_{k}(x)=c_{k}\right\},
$$

la variété affine. En utilisant la méthode décrite précédemment, on obtient, les résultats suivants [9] :

Theorem 4.1. a) Le système (4.2) dans le cas de Kowalewski admet deux familles de solutions en séries de Laurent méromorphes

$$
M=\sum_{k=0}^{\infty} M^{(k)} t^{k-1}, \Gamma=\sum_{k=0}^{\infty} \Gamma^{(k)} t^{k-2},
$$


Vol. LIII (2015) Systèmes dynamiques et géométrie

$$
\begin{aligned}
M^{(0)}+\left[M^{(0)}, \Lambda M^{(0)}\right]+\left[\Gamma^{(0)}, L\right] & =0 \\
2 \Gamma^{(0)}+\left[\Gamma^{(0)}, \Lambda M^{(0)}\right] & =0
\end{aligned}
$$

dépendant d'une variable libre $\alpha$ et définissant deux droites. Tandis que $M^{(k)}$ et $\Gamma^{(k)}$ satisfont aux systèmes linéaires

$$
\begin{gathered}
(L-k \boldsymbol{I})\left(\begin{array}{c}
M^{(1)} \\
\Gamma^{(1)}
\end{array}\right)=0, \\
(L-k \boldsymbol{I})\left(\begin{array}{c}
M^{(k)} \\
\Gamma^{(k)}
\end{array}\right)=\left\{\begin{array}{c}
-\sum_{i=1}^{k-1}\left[M^{(i)}, \Lambda M^{(k-i)}\right], \text { pour } \quad k \geq 2 \\
-\sum_{i=1}^{k-1}\left[\Gamma^{(i)}, \Lambda M^{(k-i)}\right], \text { pour } \quad k \geq 2
\end{array}\right.
\end{gathered}
$$

où L est la matrice jacobienne de (4.7). Ces systèmes fournissent une variable libre à chacun des niveaux $k=1,2,3$ et 4 . Explicitement, on a

$$
\begin{aligned}
m_{1}(t) & =\frac{\alpha}{t} \pm i\left(\alpha^{2}-2\right) \beta+o(t), & \gamma_{1}(t) & =\frac{1}{2 t^{2}}+o(t), \\
m_{2}(t) & = \pm \frac{i \alpha}{t}-\alpha^{2} \beta+o(t), & \gamma_{2}(t) & = \pm \frac{i}{2 t^{2}}+o(t), \\
m_{3}(t) & = \pm \frac{i}{t}+\alpha \beta+o(t), & \gamma_{3}(t) & =\frac{\beta}{t}+o(t) .
\end{aligned}
$$

b) Les diviseurs des pôles des fonctions $M$ et $\Gamma$ sont deux surfaces de Riemann

$$
\mathcal{D}_{\varepsilon}: \beta^{4}\left(\alpha^{2}-1\right)^{2}-\left(c_{1} \beta^{2}-2 \varepsilon c_{2} \beta-1\right)\left(\alpha^{2}-1\right)+c_{4}=0, \varepsilon^{2}=-1,
$$

irréductibles isomorphes et chacune de genre 3. Ce sont deux revêtements

$$
\varphi_{\varepsilon}: \mathcal{D}_{\varepsilon} \longrightarrow \mathcal{D}_{\varepsilon}^{0}:(\alpha, u, \beta) \longmapsto(u, \beta),
$$

doubles ramifiés en quatre points de courbes elliptiques :

$$
\mathcal{D}_{\varepsilon}^{0}: u^{2}=\left(c_{1} \beta^{2}-2 \varepsilon c_{2} \beta-1\right)^{2}-4 c_{4} \beta^{4} .
$$

c) L'espace $\mathcal{L}\left(\mathcal{D}_{\varepsilon=i}+\mathcal{D}_{\varepsilon=-i}\right)$ est engendré par les huit fonctions :

$$
\begin{aligned}
& f_{0}=1, f_{1}=m_{1}, f_{2}=m_{2}, f_{3}=m_{3}, f_{4}=\gamma_{3}, f_{5}=f_{1}^{2}+f_{2}^{2}, \\
& f_{6}=4 f_{1} f_{4}-f_{3} f_{5}, f_{7}=\left(f_{2} \gamma_{1}-f_{1} \gamma_{2}\right) f_{3}+2 f_{4} \gamma_{2} .
\end{aligned}
$$

En outre, l'application $\left(\mathcal{D}_{\varepsilon=i}+\mathcal{D}_{\varepsilon=-i}\right) \longrightarrow \mathbb{C P}^{7}$, définie par

$$
p=(\alpha, u, \beta) \longmapsto \lim _{t \rightarrow 0} t\left(1, f_{1}(p), \ldots, f_{7}(p)\right)=\left(0, f_{1}^{(0)}(p), \ldots, f_{7}^{(0)}(p)\right)
$$


plonge $\left(\mathcal{D}_{\varepsilon=i}+\mathcal{D}_{\varepsilon=-i}\right)$ dans $\mathbb{C P}^{7}$ de telle façon que $\mathcal{D}_{\varepsilon=i}$ intersecte $\mathcal{D}_{\varepsilon=-i}$ transversalement en quatre points à l'infini et que le genre géométrique de $\left(\mathcal{D}_{\varepsilon=i}+\mathcal{D}_{\varepsilon=-i}\right)$ est 9 . Les orbites du champ de vecteurs (4.4) passant à travers $\left(\mathcal{D}_{\varepsilon=i}+\mathcal{D}_{\varepsilon=-i}\right)$ forment une surface lisse $\Sigma$ tout le long du diviseur $\left(\mathcal{D}_{\varepsilon=i}+\mathcal{D}_{\varepsilon=-i}\right)$ tel que $: \Sigma \backslash\left(\mathcal{D}_{\varepsilon=i}+\mathcal{D}_{\varepsilon=-i}\right) \subset M_{c}$ (4.6). En outre, la variété $\widetilde{M}_{c}=M_{c} \cup \Sigma$, est lisse, compacte et connexe.

d) Le champ de vecteur (4.4) (4.9) est régulier le long de $\left(\mathcal{D}_{\varepsilon=i}+\mathcal{D}_{\varepsilon=-i}\right)$, transversal en tout point $\beta \neq 0($ resp. $\beta \neq \infty)$ et doublement tangent en $\beta=0$ (resp. $\beta=\infty)$. Ces champs de vecteurs se prolongent de façon holomorphe et demeurent indépendants sur la variété $\widetilde{M}_{c}$.

e) La variété $\widetilde{M}_{c}$ est une surface abélienne sur laquelle les flots hamiltoniens (4.4) et (4.5) se linéarisent. Il existe sur la surface abélienne $\widetilde{M}_{c}$ deux différentielles holomorphes $d t_{1}$ et $d t_{2}$ telles que:

$$
d t_{\left.1\right|_{\mathcal{D}_{\varepsilon}}}=\omega_{1}=\frac{k_{1}\left(\alpha^{2}-1\right) \beta^{2} d \beta}{\alpha u}, \quad d t_{\left.2\right|_{\mathcal{D}_{\varepsilon}}}=\omega_{2}=\frac{k_{2} d \beta}{\alpha u},
$$

où $k_{1}, k_{2} \in \mathbb{C}$ et $\omega_{1}, \omega_{2}$ sont des différentielles holomorphes sur $\mathcal{D}_{\varepsilon}$ (4.8). En outre, l'espace des différentielles holomorphes sur $\left(\mathcal{D}_{\varepsilon=i}+\mathcal{D}_{\varepsilon=-i}\right)$ est $\left\{f_{1}^{(0)} \omega_{2} f_{2}^{(0)} \omega_{2}, \ldots, f_{7}^{(0)} \omega_{2}\right\} \oplus\left\{\omega_{1}, \omega_{2}\right\}$ ò̀ $f_{1}^{(0)}, f_{2}^{(0)}, \ldots f_{7}^{(0)}$ sont les premiers coefficients des fonctions $f_{1}, f_{2}, \ldots, f_{7} \in \mathcal{L}\left(\mathcal{D}_{\varepsilon=i}+\mathcal{D}_{\varepsilon=-i}\right)$ (4.11) et le plongement de $\left(\mathcal{D}_{\varepsilon=i}+\mathcal{D}_{\varepsilon=-i}\right)$ dans $\mathbb{C P}^{7}$ est à deux différentielles holomorphes près le plongement canonique

$$
p=(\alpha, u, \beta) \in\left(\mathcal{D}_{\varepsilon=i}+\mathcal{D}_{\varepsilon=-i}\right) \longmapsto\left\{\omega_{2}, f_{1}^{(0)} \omega_{2}, f_{2}^{(0)} \omega_{2}, \ldots, f_{7}^{(0)} \omega_{2}\right\} \in \mathbb{C P}^{7} .
$$

f) En désignant par Prym $\left(\mathcal{D}_{\varepsilon} / \mathcal{D}_{\varepsilon}^{0}\right)$ la variété de Prym, i.e., la composante neutre de KerNm où $N m: \operatorname{Jac}\left(\mathcal{D}_{\varepsilon}\right) \rightarrow \operatorname{Jac}\left(\mathcal{D}_{\varepsilon}^{0}\right), \sum m_{i} p_{i} \mapsto \sum m_{i} \varphi\left(p_{i}\right)$ est le morphisme norme, alors la surface abélienne $\widetilde{M}_{c}$ est caractérisée comme étant la duale de la variété Prym $\left(\mathcal{D}_{\varepsilon} / \mathcal{D}_{\varepsilon}^{0}\right)$ du revêtement double $\varphi_{\varepsilon}(4.9)$ de $\mathcal{D}_{\varepsilon}(4.8) \operatorname{sur} \mathcal{D}_{\varepsilon}^{0}(4.10)$.

\subsection{Flot géodésique sur le groupe $S O(4)$ pour la métrique de Ma- nakov}

On considère le groupe $S O(4)$, son algèbre de Lie so(4) et la forme de Killing dans $s o(4):\langle X, Y\rangle=-\frac{1}{2} \operatorname{tr}(X . Y)$, où

$$
X=\left(X_{i j}\right)_{1 \leq i, j \leq 4}=\sum_{i=1}^{6} x_{i} e_{i}=\left(\begin{array}{cccc}
0 & -x_{3} & x_{2} & -x_{4} \\
x_{3} & 0 & -x_{1} & -x_{5} \\
-x_{2} & x_{1} & 0 & -x_{6} \\
x_{4} & x_{5} & x_{6} & 0
\end{array}\right) \in \operatorname{so}(4) .
$$


Une métrique invariante à gauche sur le groupe $S O(4)$ est définie par une application linéaire non-singulière $\Lambda:$ so(4) $\longrightarrow$ so(4), $X \longmapsto \Lambda X$ avec $\langle g X, g Y\rangle=\left\langle X, \Lambda^{-1} Y\right\rangle, g \in S O(4)$. Le flot géodésique pour cette métrique s'écrit sous la forme

$$
\frac{d X}{d t}=[X, \Lambda X],(\text { Equations d'Euler-Arnold })
$$

où

$$
\Lambda X=\sum_{i=1}^{6} \lambda_{i} x_{i} e_{i}=\left(\begin{array}{cccc}
0 & -\lambda_{3} x_{3} & \lambda_{2} x_{2} & -\lambda_{4} x_{4} \\
\lambda_{3} x_{3} & 0 & -\lambda_{1} x_{1} & -\lambda_{5} x_{5} \\
-\lambda_{2} x_{2} & \lambda_{1} x_{1} & 0 & -\lambda_{6} x_{6} \\
\lambda_{4} x_{4} & \lambda_{5} x_{5} & \lambda_{6} x_{6} & 0
\end{array}\right) \in \operatorname{so}(4)
$$

Comme pour so (3), en utilisant l'isomorphisme entre $(s o(4),[]$,$) et \left(\mathbb{R}^{6}, \wedge\right)$, les équations (4.12) s'écrivent explicitement sous la forme

$$
\begin{aligned}
\frac{d x_{1}}{d t} & =\left(\lambda_{3}-\lambda_{2}\right) x_{2} x_{3}+\left(\lambda_{6}-\lambda_{5}\right) x_{5} x_{6}, \\
\frac{d x_{2}}{d t} & =\left(\lambda_{1}-\lambda_{3}\right) x_{1} x_{3}+\left(\lambda_{4}-\lambda_{4}\right) x_{4} x_{6}, \\
\frac{d x_{3}}{d t} & =\left(\lambda_{2}-\lambda_{1}\right) x_{1} x_{2}+\left(\lambda_{5}-\lambda_{4}\right) x_{4} x_{5}, \\
\frac{d x_{4}}{d t} & =\left(\lambda_{3}-\lambda_{5}\right) x_{3} x_{5}+\left(\lambda_{6}-\lambda_{2}\right) x_{2} x_{6}, \\
\frac{d x_{5}}{d t} & =\left(\lambda_{4}-\lambda_{3}\right) x_{3} x_{4}+\left(\lambda_{1}-\lambda_{6}\right) x_{1} x_{6}, \\
\frac{d x_{6}}{d t} & =\left(\lambda_{2}-\lambda_{4}\right) x_{2} x_{4}+\left(\lambda_{5}-\lambda_{1}\right) x_{1} x_{5} .
\end{aligned}
$$

Ces équations forment un champ de vecteurs hamiltonien

$$
\frac{d x(t)}{d t}=J \frac{\partial H}{\partial x}, x \in \mathbb{R}^{6}
$$

avec

$$
H=\frac{1}{2}\langle X, \Lambda X\rangle=\frac{1}{2}\left(\lambda_{1} x_{1}^{2}+\lambda_{2} x_{2}^{2}+\cdots+\lambda_{6} x_{6}^{2}\right),
$$

et

$$
J=\left(\begin{array}{cccccc}
0 & -x_{3} & x_{2} & 0 & -x_{6} & x_{5} \\
x_{3} & 0 & -x_{1} & x_{6} & 0 & -x_{4} \\
-x_{2} & x_{1} & 0 & -x_{5} & x_{4} & 0 \\
0 & -x_{6} & x_{5} & 0 & -x_{3} & x_{2} \\
x_{6} & 0 & -x_{4} & x_{3} & 0 & -x_{1} \\
-x_{5} & x_{4} & 0 & -x_{2} & x_{1} & 0
\end{array}\right) \in s o(6)
$$


Le problème en question admet les quatre intégrales premières suivantes :

$$
\begin{aligned}
H_{1} & \equiv H=\frac{1}{2}\langle X, \Lambda X\rangle=\frac{1}{2}\left(\lambda_{1} x_{1}^{2}+\lambda_{2} x_{2}^{2}+\cdots+\lambda_{6} x_{6}^{2}\right), \\
H_{2} & =\frac{1}{2}\left(x_{1}^{2}+x_{2}^{2}+\cdots+x_{6}^{2}\right), \\
H_{3} & =x_{1} x_{4}+x_{2} x_{5}+x_{3} x_{6}, \\
H_{4} & =\frac{1}{2}\left(\mu_{1} x_{1}^{2}+\mu_{2} x_{2}^{2}+\cdots+\mu_{6} x_{6}^{2}\right) .
\end{aligned}
$$

Les paramètres $\lambda_{1}, \ldots, \lambda_{6}$ satisfont à la condition de Manakov [16] :

$$
\begin{array}{lll}
\lambda_{1}=\frac{\beta_{2}-\beta_{3}}{\alpha_{2}-\alpha_{3}}, & \lambda_{2}=\frac{\beta_{1}-\beta_{3}}{\alpha_{1}-\alpha_{3}}, & \lambda_{3}=\frac{\beta_{1}-\beta_{2}}{\alpha_{1}-\alpha_{2}}, \\
\lambda_{4}=\frac{\beta_{1}-\beta_{4}}{\alpha_{1}-\alpha_{4}}, & \lambda_{5}=\frac{\beta_{2}-\beta_{4}}{\alpha_{2}-\alpha_{4}}, & \lambda_{6}=\frac{\beta_{3}-\beta_{4}}{\alpha_{3}-\alpha_{4}},
\end{array}
$$

avec $\alpha_{i}, \beta_{i} \in \mathbb{C}, \prod_{i<j}\left(\alpha_{i}-\beta_{j}\right) \neq 0$. Les paramètres $\mu_{1}, \ldots, \mu_{6}$ peuvent avoir une paramétrisation similaire à celle de $\lambda_{1}, \ldots, \lambda_{6}$.

Nous allons montrer (pour le détail voir $[1,3,6]$ ) que le problème en question est algèbriquement complètement intégrable et qu'en outre la linéarisation s'effectue sur une variété de Prym. La variété invariante

$$
M_{c}=\bigcap_{i=1}^{4}\left\{x: H_{i}(x)=c_{i}\right\} \subset \mathbb{C}^{6},
$$

où $c_{1}, c_{2}, c_{3}, c_{4}$ sont des constantes génériques, est une surface affine lisse et le problème ici consiste aussi à complèter $M_{c}$ en une surface abélienne $\widetilde{M}_{c}$. Le système des équations différentielles (4.13) ou (4.14) possède des solutions sous la forme de séries de Laurent,

$$
x(t)=t^{-1}\left(x^{(0)}+x^{(1)} t+x^{(2)} t^{2}+\ldots\right),
$$

dépendant de $\operatorname{dim}$ (espace de phase) $-1=5$ paramètres libres. Considérons l'ensemble

$$
\begin{aligned}
\mathcal{D}= & \bigcap_{i=1}^{4}\left\{\text { coefficient de } t^{0} \text { dans } H_{i}(x(t))=c_{i}\right\}, \\
= & \text { une surface de Riemann d'équation affine : } \\
& w^{2}+F\left(x_{4}^{(0)}, x_{5}^{(0)}, x_{6}^{(0)}\right)=0,
\end{aligned}
$$

où $w$ est un paramètre arbitraire,

$$
F\left(x_{4}^{(0)}, x_{5}^{(0)}, x_{6}^{(0)}\right) \equiv c_{1}\left(x_{5}^{(0)} x_{6}^{(0)}\right)^{2}+c_{2}\left(x_{4}^{(0)} x_{6}^{(0)}\right)^{2}+c_{3}\left(x_{4}^{(0)} x_{5}^{(0)}\right)^{2}+c_{4} x_{4}^{(0)} x_{5}^{(0)} x_{6}^{(0)},
$$


et où $x_{4}^{(0)}, x_{5}^{(0)}, x_{6}^{(0)}$ paramètrisent la courbe elliptique

$$
\mathcal{E}:\left\{\begin{aligned}
\left(x_{4}^{(0)}\right)^{2}+\left(x_{5}^{(0)}\right)^{2}+\left(x_{6}^{(0)}\right)^{2} & =0 \\
\left(\beta x_{5}^{(0)}+\alpha x_{6}^{(0)}\right)\left(\beta x_{5}^{(0)}-\alpha x_{6}^{(0)}\right) & =1
\end{aligned}\right.
$$

avec $\alpha, \beta$ tels que : $\alpha^{2}+\beta^{2}+1=0$. La courbe $\mathcal{D}$ est un revêtement double ramifié le long de $\mathcal{E}$. Les points de branchements sont les 16 zéros de $F\left(x_{4}^{(0)}, x_{5}^{(0)}, x_{6}^{(0)}\right)$ sur $\mathcal{E}$. Comme la courbe $\mathcal{D}$ n'est pas ramifiée à l'infini, alors d'après la formule de Riemann-Hurwitz le genre de $\mathcal{D}$ est 9 . Par conséquent, on a le résultat suivant $[1,6]$ :

Theorem 4.2. Le système différentiel (4.14) est algébriquement complètement intégrable et le flot correspondant évolue sur une surface abélienne $\widetilde{M}_{c} \cong \mathbb{C}^{2} / L_{\Omega}$ où le réseau $L_{\Omega}$ est engendré par la matrice des périodes

$$
\Omega=\left(\begin{array}{llll}
2 & 0 & a & c \\
0 & 4 & c & b
\end{array}\right), \quad \operatorname{Im}\left(\begin{array}{ll}
a & c \\
c & b
\end{array}\right)>0, \quad(a, b, c \in \mathbb{C}) .
$$

La surface affine $M_{c}(4.15)$ se complète en $\widetilde{M}_{c}$ par l'adjonction d'une courbe lisse $\mathcal{D}$ (4.16) de genre 9, laquelle est un revêtement ramifié le long d'une courbe elliptique $\mathcal{E}$ (4.17).

En posant $\zeta \equiv \alpha^{2}$, la courbe $\mathcal{D}$ peut-être vue comme étant un revêtement de degré 4 non ramifié le long de la courbe de genre 3 suivante :

$$
\mathcal{C}: F(\theta, \zeta) \equiv\left[\theta^{2}+c_{1} \beta^{2} \gamma^{2}+\left(c_{2} \gamma^{2}+c_{3} \beta^{2}\right) \zeta\right]^{2}-c_{4}^{2} \zeta \beta^{2} \gamma^{2}=0
$$

Notons que l'application $\sigma: \mathcal{C} \longrightarrow \mathcal{C},(\theta, \zeta) \longmapsto(-\theta, \zeta)$, est une involution sur $\mathcal{C}$ et que le quotient $\mathcal{C}_{0}=\mathcal{C} / \sigma$ est une courbe elliptique définie par

$$
\mathcal{C}_{0}: \eta^{2}=c_{4}^{2} \zeta\left(d_{1}^{2} \zeta-1\right)\left(d_{2}^{2} \zeta+1\right)
$$

La courbe $\mathcal{C}$ est un revêtement double ramifié le long de $\mathcal{C}_{0}$

$$
\begin{gathered}
\mathcal{\longrightarrow} \longrightarrow \mathcal{C}_{0}, \quad(\theta, \eta, \zeta) \longmapsto(\eta, \zeta), \\
\mathcal{C}: \begin{cases}\theta^{2} & =-c_{1} \beta^{2} \gamma^{2}-\left(c_{2} \gamma^{2}+c_{3} \beta^{2}\right) \zeta+\eta, \\
\eta^{2} & =c_{4}^{2} \zeta\left(d_{1}^{2} \zeta-1\right)\left(d_{2}^{2} \zeta+1\right) .\end{cases}
\end{gathered}
$$

On montre [6] que :

Theorem 4.3. La surface abélienne $\widetilde{M}_{c}$ peut-être identifiée à la variété de $\operatorname{Prym} \operatorname{Prym}\left(\mathcal{C} / \mathfrak{C}_{0}\right)$ du revêtement $(4.18)$ et le problème se linéarise sur cette variété. 


\subsection{Le système différentiel de Hénon-Heiles}

Les équations différentielles non-linéaires de Hénon-Heiles [7] sont définies par

$$
\begin{array}{llrl}
\frac{d y_{1}}{d t} & =x_{1}, & \frac{d x_{1}}{d t} & =-A y_{1}-2 y_{1} y_{2}, \\
\frac{d y_{2}}{d t} & =x_{2}, & \frac{d x_{2}}{d t} & =-B y_{2}-y_{1}^{2}-\varepsilon y_{2}^{2}
\end{array}
$$

où $A, B$ sont des constantes. On rencontre ce système dans plusieurs problèmes d'applications; notamment en dynamique stellaire, en mécanique statistique ainsi qu'en mécanique quantique. Il fournit un modèle pour le mouvement d'une étoile dans une galaxie cylindrique ainsi que pour les oscillations des atomes dans une molécule. Le système (4.19) admet les intégrales premières suivantes :

$$
\begin{aligned}
& H_{1}=\frac{1}{2}\left(x_{1}^{2}+x_{2}^{2}+A y_{1}^{2}+B y_{2}^{2}\right)+y_{1}^{2} y_{2}+6 y_{2}^{3} \\
& H_{2}=y_{1}^{4}+4 y_{1}^{2} y_{2}^{2}-4 x_{1}\left(x_{1} y_{2}-x_{2} y_{1}\right)+4 A y_{1}^{2} y_{2}+(4 A-B)\left(x_{1}^{2}+A y_{1}^{2}\right)
\end{aligned}
$$

Adler et van Moerbeke ont montré que ce cas est lié par une transformation birationnelle au problème de Kowalewski ainsi qu'au flot géodésique sur $S O(4)$ pour une métrique de Manakov. On peut réecrire les équations (4.19) sous la forme d'un champ de vecteurs hamiltonien $\frac{d x}{d t}=J \frac{\partial H}{\partial x}, J=\left(\begin{array}{cc}0 & -I \\ I & 0\end{array}\right)$ où $x=\left(y_{1}, y_{2}, x_{1}, x_{2}\right), H \equiv H_{1}$ (4.20) est l'hamiltonien et $I$ est la matrice unité. La fibre $M_{c}$ définie par l'intersection des intégrales premières du système différentiel de Hénon-Heiles se complète en une surface abélienne $\widetilde{M}_{c}$, par l'adjonction d'une surface de Riemann $\mathcal{D}$ lisse hyperelliptique de genre 3 ; laquelle est un revêtement double ramifié le long d'une courbe elliptique $\mathcal{E}$. Aussi $\widetilde{M}_{c}$ peut être identifiée à la duale d'une variété de Prym $\operatorname{Prym}^{\vee}(\mathcal{D} / \mathcal{E})$ et le système se linéarise sur cette variété. Plus précisément, on a [10] :

Theorem 4.4. a) Le système (4.19) admet une famille de solutions de Laurent méromorphes dépendant de trois paramètres libres $\alpha, \beta, \gamma$ :

$$
\begin{array}{r}
y_{1}=\frac{\alpha}{t}+\left(\frac{\alpha^{3}}{12}+\frac{\alpha A}{2}-\frac{\alpha B}{12}\right) t+\beta t^{2}+y_{1}^{(4)} t^{3}+y_{1}^{(5)} t^{4}+y_{1}^{(6)} t^{5}+\cdots \\
y_{2}=-\frac{1}{t^{2}}+\frac{\alpha^{2}}{12}-\frac{B}{12}+\left(\frac{\alpha^{4}}{48}+\frac{\alpha^{2} A}{10}-\frac{\alpha^{2} B}{60}-\frac{B^{2}}{240}\right) t^{2}+\frac{\alpha \beta}{3} t^{3}+\gamma t^{4}+\cdots
\end{array}
$$


où

$$
\begin{aligned}
y_{1}^{(4)}= & \frac{\alpha A B}{24}-\frac{\alpha^{5}}{72}+\frac{11 \alpha^{3} B}{720}-\frac{11 \alpha^{3} A}{120}-\frac{\alpha B^{2}}{720}-\frac{\alpha A^{2}}{8}, \\
y_{1}^{(5)}= & -\frac{\beta \alpha^{2}}{12}+\frac{\beta B}{60}-\frac{A \beta}{10}, \\
y_{1}^{(6)}= & -\frac{\alpha \gamma}{9}-\frac{\alpha^{7}}{15552}-\frac{\alpha^{5} A}{2160}+\frac{\alpha^{5} B}{12960}+\frac{\alpha^{3} B^{2}}{25920}+\frac{\alpha^{3} A^{2}}{1440} \\
& -\frac{\alpha^{3} A B}{4320}+\frac{\alpha A B^{2}}{1440}-\frac{\alpha B^{3}}{19440}-\frac{\alpha A^{2} B}{288}+\frac{\alpha A^{3}}{144},
\end{aligned}
$$

Le diviseur des pôles des fonctions $x=\left(y_{1}, y_{2}, x_{1}, x_{2}\right)$ est une surface de Riemann lisse hyperelliptique de genre 3 :

$$
\mathcal{D}: a_{1} \beta^{2}+a_{2} \alpha^{8}+a_{3} \alpha^{6}+a_{4} \alpha^{4}+a_{5} \alpha^{2}+a_{6}=0,
$$

ò̀ $a_{1}=36, a_{2}=\frac{7}{432}, a_{3}=\frac{5}{12} A-\frac{13}{216} B, a_{4}=\frac{671}{15120} B^{2}+\frac{17}{7} A^{2}-\frac{943}{1260} B A, a_{5}=$ $\frac{2}{9} A B^{2}-\frac{1}{2520} B^{3}-\frac{10}{7} c_{1}-\frac{13}{6} A^{2} B+4 A^{3}, a_{6}=-c_{2}$. C'est un revêtement double ramifié en quatre points d'une courbe elliptique

$$
\mathcal{E}: a_{1} \beta^{2}+a_{2} \zeta^{4}+a_{3} \zeta^{3}+a_{4} \zeta^{2}+a_{5} \zeta+a_{6}=0 .
$$

b) Soit $M_{c}$ la fibre définie par

$$
M_{c}=\bigcap_{i=1}^{2}\left\{x: H_{i}(x)=c_{i}\right\} \subset \mathbb{C}^{4}
$$

où $\left(c_{1}, c_{2}\right) \in \mathbb{C}^{2}$ n'est pas une valeur critique. Alors, les orbites du champ de vecteurs (4.19) passant à travers $\mathcal{D}$ forment une surface lisse $\Sigma$ tout le long de $\mathcal{D}$ tel que $: \Sigma \backslash \mathcal{D} \subset M_{c}$. En outre, la variété $\widetilde{M}_{c}=M_{c} \cup \Sigma$ est lisse, compacte et connexe. La variété $\widetilde{M}_{c}$ est munie de deux champs de vecteurs commutant et linéairement indépendants en chaque point. Ces champs se prolongent de façon holomorphe et demeurent indépendants sur $\widetilde{M}_{c}$. Le système différentiel (4.19) est algébriquement complètement intégrable et le flot correspondant évolue sur une surface abélienne $\widetilde{M}_{c} \simeq \mathbb{C}^{2} /$ réseau.

c) Le diviseur $2 \mathcal{D}$ détermine sur $\widetilde{M}_{c}$ une polarization du type $(2,4)$. Autrement dit, on a $M_{c}=\left\{\begin{array}{l}\text { surface abélienne } \widetilde{M}_{c} \simeq \mathbb{C}^{2} / L_{\Omega} \text { où } L_{\Omega} \text { est le réseau engendré par } \\ \text { les périodes de la matrice }\left(\begin{array}{llll}2 & 0 & a & b \\ 0 & 4 & b & c\end{array}\right), \quad \operatorname{Im}\left(\begin{array}{ll}a & b \\ b & c\end{array}\right)>0\end{array}\right\}$, $\backslash$ courbe algébrique $2 \mathcal{D}$ de genre 9 tracée sur $\left.\widetilde{M}_{c}\right\}$. 
La surface abélienne $\widetilde{M}_{c}$ qui complète la fibre $M_{c}$ (4.23) peut être identifiée à la duale d'une variété de Prym Prym ${ }^{\vee}(\mathcal{D} / \mathcal{E})$ du revêtement double $: \mathcal{D} \longrightarrow$ $\mathcal{E},(\alpha, \beta) \longmapsto(\zeta, \beta)$ où $\mathcal{D}$ est la surface de Riemann hyperelliptique de genre 3 d'équation (4.21) et $\mathcal{E}$ la courbe elliptique d'équation (4.22). En outre, le système différentiel de Hénon-Heiles se linéarise sur cette variété.

\subsection{Potentiel quartique, système de Garnier}

Considérons le hamiltonien

$$
H=\frac{1}{2}\left(x_{1}^{2}+x_{2}^{2}\right)-\frac{1}{2}\left(\lambda_{1} y_{1}^{2}+\lambda_{2} y_{2}^{2}\right)+\frac{1}{4}\left(y_{1}^{2}+y_{2}^{2}\right)^{2},
$$

où $\lambda_{1}$ et $\lambda_{2}$ sont des constantes. Le système de Garnier correspondant est donné par

$$
\begin{aligned}
\frac{d y_{1}}{d t}=x_{1}, & \frac{d x_{1}}{d t}=\left(\lambda_{1}-y_{1}^{2}-y_{2}^{2}\right) y_{1}, \\
\frac{d y_{2}}{d t}=x_{2}, & \frac{d x_{2}}{d t}=\left(\lambda_{2}-y_{2}^{2}-y_{1}^{2}\right) y_{2} .
\end{aligned}
$$

Soit $M_{c}$ la fibre définie par $M_{c}=\bigcap_{i=1}^{2}\left\{x: H_{i}(x)=c_{i}\right\} \subset \mathbb{C}^{4}$ où

$$
\begin{aligned}
H_{1}= & \frac{1}{2}\left(x_{1}^{2}+x_{2}^{2}\right)-\frac{1}{2}\left(\lambda_{1} y_{1}^{2}+\lambda_{2} y_{2}^{2}\right)+\frac{1}{4}\left(y_{1}^{2}+y_{2}^{2}\right)^{2}, \\
H_{2}= & \frac{1}{4}\left(\left(x_{1} y_{2}-x_{2} y_{1}\right)^{2}-\left(\lambda_{2} y_{1}^{4}+\lambda_{1} y_{2}^{4}\right)-\left(\lambda_{1}+\lambda_{2}\right) y_{1}^{2} y_{2}^{2}\right) \\
& +\frac{1}{2}\left(\lambda_{1} \lambda_{2}\left(y_{1}^{2}+y_{2}^{2}\right)-\left(\lambda_{2} x_{1}^{2}+\lambda_{1} x_{2}^{2}\right)\right) .
\end{aligned}
$$

avec $c_{1}, c_{2}$ des constantes génériques et $\lambda_{1} \neq \lambda_{2}$. En utilisant la méthode décrite précédemment, on obtient pour ce problème les résultats suivants :

Theorem 4.5. a) La variété $\widetilde{M}_{c}$ qui complète la fibre $M_{c}$ est une variété kählérienne munie d'une métrique hermitienne de la forme

$$
d s^{2}=d \tau_{1} \otimes d \bar{\tau}_{1}+d \tau_{2} \otimes d \bar{\tau}_{2}, \quad\left(\tau_{1}, \tau_{2}\right) \in \mathbb{C} .
$$

La surface invariante $M_{c}$ forme la partie affine d'une surface abélienne $\widetilde{M}_{c}$ avec $\widetilde{M}_{c} \backslash M_{c}=\mathcal{D}$, où $\mathcal{D} \equiv \mathcal{C}_{1}+\mathcal{C}_{-1}$ a deux composantes $\mathcal{C}_{1}$ et $\mathcal{C}_{-1}$, chacune est une surface de Riemann de genre 3 ayant même équation affine

$$
\mathcal{C}_{ \pm 1}: \beta^{2}=\left(2+\alpha^{2}\right)\left(A_{1} \alpha^{6}+A_{2} \alpha^{4}+A_{3} \alpha^{2}+A_{4}\right)
$$


ò̀ $\lambda \equiv \lambda_{1}-\lambda_{2}, A_{1} \equiv \lambda^{3} / 72, A_{2} \equiv \lambda^{2}\left(2 \lambda_{1}-\lambda_{2}\right) / 36, A_{3} \equiv \lambda\left(\lambda \lambda_{1}-c_{1}\right) / 18$, $A_{4} \equiv-\left(\lambda_{1} c_{1}+c_{2}\right) / 9$. L'application $\sigma: \mathcal{C}_{ \pm 1} \longrightarrow \mathcal{C}_{ \pm 1},(\alpha, \beta) \longmapsto(-\alpha, \beta)$, est une involution sur $\mathcal{C}_{ \pm 1}$. La surface de Riemann $\mathcal{C}_{ \pm 1}$ est un revêtement double $\mathcal{C}_{ \pm 1} \longrightarrow \mathcal{E},(\alpha, \beta) \longmapsto(\zeta, \beta)$, ramifié en quatre points d'une courbe elliptique :

$$
\mathcal{E}: \beta^{2}=(2+\zeta)\left(A_{1} \zeta^{3}+A_{1} \zeta^{2}+A_{3} \zeta+A_{4}\right) .
$$

En outre, la surface $\mathcal{C}_{ \pm 1}$ peut-être vue comme étant un revêtement double $\mathcal{C}_{ \pm 1} \longrightarrow \mathcal{H},(\alpha, \beta) \longmapsto(\zeta, \eta)$, non ramifié d'une surface de Riemann hyperelliptique $\mathcal{H}$ de genre 2 :

$$
\mathcal{H}: \eta^{2}=\zeta(2+\zeta)\left(A_{1} \zeta^{3}+A_{1} \zeta^{2}+A_{3} \zeta+A_{4}\right) .
$$

Les flots engendrés par $H_{1}$ et $H_{2}$ sont des lignes droites sur $\widetilde{M}_{c}$.

b) Les 16 fonctions

$$
\begin{aligned}
f_{0} & =1, f_{1}=y_{1}, f_{2}=y_{2}, f_{3}=x_{1}, f_{4}=x_{2}, f_{5}=f_{1}^{2}, f_{6}=f_{2}^{2}, f_{7}=f_{1} f_{2}, \\
f_{8} & =f_{1} f_{4}-f_{2} f_{3}, f_{9}=f_{1} f_{8}, f_{10}=f_{2} f_{8}, f_{11}=f_{8}^{2}, \\
f_{12} & =\left(f_{5}+f_{6}\right) f_{8}-2\left(\lambda_{1} f_{1} f_{4}-\lambda_{2} f_{2} f_{3}\right), f_{13}=\left(f_{5}+f_{6}\right) f_{1} f_{2}+2 f_{3} f_{4}, \\
f_{14} & =f_{3} f_{8}-\left(\lambda_{2}-\lambda_{1}\right) f_{1} f_{7}, f_{15}=f_{4} f_{8}-\left(\lambda_{2}-\lambda_{1}\right) f_{2} f_{7},
\end{aligned}
$$

forment une base de l'espace vectoriel $\mathcal{L}(2 \mathcal{D})$ des fonctions holomorphes sur $\widetilde{M}_{c}$ et ayant au plus un pôle double le long de $\mathcal{D}$. A l'aide de ces fonctions on plonge $\mathcal{C}_{+1}$ et $\mathcal{C}_{-1}$ dans l'espace projectif $\mathbb{C P}^{15}$ et on montre que ces surfaces de Riemann ont deux points en commun en lequel chacune est tangente à l'autre. Le diviseur $\mathcal{D} \equiv \mathcal{C}_{1}+\mathcal{C}_{-1}$ (resp. $\left.2 \mathcal{D}\right)$ ainsi obtenu a un genre égal à 5 (resp. 17). En outre, l'application

$$
\widetilde{M}_{c} \simeq \mathbb{C}^{2} / \text { Lattice } \longrightarrow \mathbb{C P}^{15},\left(t_{1}, t_{2}\right) \longmapsto\left[\left(1, f_{1}\left(t_{1}, t_{2}\right), \ldots, f_{15}\left(t_{1}, t_{2}\right)\right)\right],
$$

est un plongement de $\widetilde{M}_{c}$ dans $\mathbb{C P}^{15}$.

\subsection{Equations couplées non-linéaires de Schrödinger}

Les équations couplées non linéaires de Schrödinger s'écrivent :

$$
\begin{aligned}
& i \frac{\partial a}{\partial z}+\frac{\partial^{2} a}{\partial t^{2}}+\Omega_{0} a+\frac{2}{3}\left(|a|^{2}+|b|^{2}\right) a+\frac{1}{3}\left(a^{2}+b^{2}\right) \bar{a}=0 \\
& i \frac{\partial b}{\partial z}+\frac{\partial^{2} b}{\partial t^{2}}-\Omega_{0} b+\frac{2}{3}\left(|a|^{2}+|b|^{2}\right) b+\frac{1}{3}\left(a^{2}+b^{2}\right) \bar{b}=0 .
\end{aligned}
$$

Les fonctions $a(z, t)$ et $b(z, t)$ dépendent des variables $z$ et $t$, la notation "-" désigne l'opérateur de conjugaison complexe, "||" désigne le module et enfin $\Omega_{0}$ est une constante. On cherche les solutions sous la forme :

$$
a(z, t)=y_{1}(t) \exp (i \Omega z), \quad b(z, t)=y_{2}(t) \exp (i \Omega z),
$$


où $y_{1}(t)$ et $y_{2}(t)$ sont deux fonctions et $\Omega$ une constante arbitraire. Dès lors, on obtient un système de deux équations différentielles non-linéaires de second ordre :

$$
\frac{d^{2} y_{1}}{d t^{2}}+\left(y_{1}^{2}+y_{2}^{2}\right) y_{1}=\left(\Omega-\Omega_{0}\right) y_{1}, \quad \frac{d^{2} y_{2}}{d t^{2}}+\left(y_{1}^{2}+y_{2}^{2}\right) y_{2}=\left(\Omega+\Omega_{0}\right) y_{2} .
$$

Ce dernier s'écrit sous la forme du système hamiltonien (4.25) avec $\lambda_{1}=$ $\Omega-\Omega_{0}, \lambda_{2}=\Omega+\Omega_{0}$ et il suffit d'appliquer les résultats obtenus dans cette partie (voir [11]).

\subsection{Champ de Yang-Mills}

Soit $F_{k l}$ le champ de Yang-Mills dans l'algèbre de Lie $T_{e} S U(2)$ du groupe $S U(2)$. C'est une expression locale du champ de Jauge ou connexion $A_{k}$ définissant la dérivée covariante de $F_{k l}$ à l'aide de l'expression :

$$
\nabla_{k} F_{k l}=\frac{\partial F_{k l}}{\partial \tau_{k}}+\left[A_{k}, F_{k l}\right]=0, F_{k l}, A_{k} \in T_{e} S U(2), 1 \leq k, l \leq 4,
$$

dans laquelle $\left[A_{k}, F_{k l}\right]$ est le crochet des deux champs dans l'algèbre de Lie du groupe de Lie $S U(2)$ et $F_{k l}=\frac{\partial A_{l}}{\partial \tau_{k}}-\frac{\partial A_{k}}{\partial \tau_{l}}+\left[A_{k}, A_{l}\right]$. Dans le cas qui nous intéresse, on a $\frac{\partial A_{l}}{\partial \tau_{k}}=0(k \neq 1), A_{1}=A_{2}=0, A_{3}=n_{1} U_{1} \in \operatorname{su}(2)$, $A_{4}=n_{2} U_{2} \in$ su $(2)$, où $n_{1}=\left[n_{2},\left[n_{1}, n_{2}\right]\right], n_{2}=\left[n_{1},\left[n_{2}, n_{1}\right]\right]$, engendrent $s u(2)$ et les équations de Yang-Mills deviennent

$$
\frac{\partial^{2} U_{1}}{\partial t^{2}}+U_{1} U_{2}^{2}=0, \quad \frac{\partial^{2} U_{2}}{\partial t^{2}}+U_{2} U_{1}^{2}=0,
$$

avec $t=\tau_{1}$. En posant $U_{1}=q_{1}, U_{2}=q_{2}, \frac{\partial U_{1}}{\partial t}=p_{1}, \frac{\partial U_{2}}{\partial t}=p_{2}$, les équations de Yang-Mills s'écrivent sous la forme d'un champ de vecteurs hamiltonien

$$
\frac{d x}{d t}=J \frac{\partial H}{\partial x}, \quad x=\left(q_{1}, q_{2}, p_{1}, p_{2}\right)^{\top}, \quad J=\left(\begin{array}{cc}
0 & -I \\
I & 0
\end{array}\right)
$$

où $H=\frac{1}{2}\left(p_{1}^{2}+p_{2}^{2}+q_{1}^{2} q_{2}^{2}\right)$. Ce système hamiltonian joue un rôle important en théorie des champs. En utilisant la transformation symplectique $p_{1}=$ $\frac{\sqrt{2}}{2}\left(x_{1}+x_{2}\right), q_{1}=\frac{1}{2}(\sqrt[4]{2})^{3}\left(y_{1}+i y_{2}\right), p_{2}=\frac{\sqrt{2}}{2}\left(x_{1}-x_{2}\right), q_{2}=\frac{1}{2}(\sqrt[4]{2})^{3}\left(y_{1}-\right.$ $\left.i y_{2}\right)$, on réecrit le hamiltonien ci-dessus sous la forme $H=\frac{1}{2}\left(x_{1}^{2}+x_{2}^{2}\right)+$ $\frac{1}{4}\left(y_{1}^{2}+y_{2}^{2}\right)^{2}$, lequel coincide avec (4.24) pour $\lambda_{1}=\lambda_{2}=0$. Et il suffit d'appliquer les résultats obtenus pour le système de Garnier. Pour de plus amples informations, on pourra consulter avec profit [14]. 


\section{Acknowldegments}

Je remercie le referee pour ses remarques et suggestions.

\section{Références}

[1] M. Adler and P. van Moerbeke, The algebraic complete integrability of geodesic flow on SO(4), Invent. Math., 67, (1982), 297-331

[2] M. Adler and P. van Moerbeke, The complex geometry of the KowalewskiPainlevé analysis, Invent. Math., 7, (1989), 3-51

[3] M. Adler, P. van Moerbeke, and P. Vanhaecke, Algebraic integrability, Painlevé geometry and Lie algebras. A series of modern surveys in mathematics, 47, (2004)

[4] V.I. Arnold, Mathematical methods in classical mechanics, Springer-Verlag, BerlinHeidelberg- New York, 1978

[5] P.A. Griffiths and J. Harris, Wiley-Interscience, New York, 1978

[6] L. Haine, Geodesic flow on $S O(4)$ and Abelian surfaces, Math. Ann., 263, (1983), $435-472$

[7] M. Hénon and C. Heiles, The applicability of the third integral of motion; some numerical experiments, Astron. J., 69, (1964), 73-79

[8] S. Kowalewski, Sur le problème de la rotation d'un corps solide autour d'un point fixe, Acta Math., 12, (1989), 177-232

[9] A. Lesfari, Abelian surfaces and Kowalewski's top, Ann. Sci. École Norm. Sup. Paris, 21, (1988), 193-223

[10] A. Lesfari, Le système différentiel de Hénon-Heiles et les variétés Prym, Pacific J. Math., 212, (2003), 125-132

[11] A. Lesfari, Équations couplées non-linéaires de Schrödinger, Afr. Diaspora J. Math., 10, (2010), 96-108

[12] A. Lesfari, Systèmes hamiltoniens complètement intégrables, Aequat. Math., 82, (2011), 165-200

[13] A. Lesfari, Etude des équations stationnaire de Schrödinger, intégrale de GelfandLevitan et de Korteweg-de-Vries, Solitons et méthode de la diffusion inverse, Aequat. Math., 85, (2013), 243-272

[14] A. Lesfari, Champ de Yang-Mills avec groupe de jauge SU(2), Mathematical Reports, 17(67), (2015), 133-153

[15] A. Lesfari, Introduction à la géométrie algébrique complexe, Hermann, Paris, 2015

[16] S.V. Manakov, Remarks on the integrals of the Euler equations of the $n$-dimensional heavy top, Func. Anal. Appl., 10, (1976), 93-94

[17] P. van Moerbeke and D. Mumford, The spectrum of difference operators and algebraic curves, Acta Math., 143, (1979), 93-154

[18] B.G. Moishezon, On $n$-dimensional compact varieties with $n$ algebraically independent meromorphic functions, Amer. Math. Soc. Transl., 63, (1967), 51-177 
[19] D. Mumford, Tata lectures on thêta II, Progress in Math., Birkhäuser, Boston, 1982

[20] P. Vanhaecke, Integrable systems in the realm of algebraic geometry, Lecture Notes in Math., 1638, Springer-Verlag, 2001
A. Lesfari
Department of Mathematics
Faculty of Sciences
University of Chouaïb Doukkali
B.P. 20, El Jadida
Morocco
E-mail: lesfariahmed@yahoo.fr

Received: 14.02.2015

Accepted: 18.05.2015 